\title{
MeDUsA: A novel system for automated axon quantification to evaluate
}

4 Yohei Nitta $^{1 *}$, Hiroki Kawai ${ }^{*}$, Jiro Osaka ${ }^{3}$, Satoko Hakeda-Suzuki ${ }^{3}$, Yoshitaka Nagai ${ }^{4}$,

$5 \quad$, Karolína Doubkováa ${ }^{6,7}$, Takashi Suzuki ${ }^{3}$, Gaia Tavosanis ${ }^{6,7}$, Atsushi Sugie ${ }^{1}$

6

7 1. Brain Research Institute, Niigata University, Niigata, Japan

8 2. LPIXEL Inc., Tokyo, Japan

9 3. School of Life Science and Technology, Tokyo Institute of Technology, Yokohama, 10 Japan

11 4. Department of Neurotherapeutics, Osaka University Graduate School of Medicine,

12 Suita, Japan

13 5. Department of Neurology, Kindai University Faculty of Medicine, Osaka-Sayama,

14 Japan

15 6. German Center for Neurodegenerative Diseases (DZNE), Bonn, Germany

16 7. LIMES Institute, University of Bonn, Germany

17

$18 *$ Equal contribution

19

20 Corresponding author: atsushi.sugie@bri.niigata-u.ac.jp (A.S.).

22 Abstract:

23 Background: Drosophila is an excellent model organism for studying human

24 neurodegenerative diseases (NDs), and the rough eye phenotype (REP) assay is a 
convenient experimental system for analysing the toxicity of ectopically expressed human disease genes. However, the association between REP and axonal degeneration,

27 an early sign of ND, remains unclear. To address this question, we developed a method

28 to evaluate axonal degeneration by quantifying the number of retinal R7 axons in

29 Drosophila; however, it requires expertise and is time-consuming. Therefore, there is a need for an easy-to-use software that can automatically quantify the axonal degeneration.

31 Result: We created MeDUsA (a 'method for the quantification of degeneration using fly axons'), which is a standalone executable computer program based on Python that combines a pre-trained deep-learning masking tool with an axon terminal counting tool.

This software automatically quantifies the number of axons from a confocal z-stack image series. Using this software, we have demonstrated for the first time directly that axons degenerate when the causative factors of NDs ( $\alpha$ Syn, Tau, TDP-43, HTT) were expressed in the Drosophila eye. Furthermore, we compared axonal toxicity of the representative causative genes of NDs and their pathological alleles with REP and found no significant correlation between them.

40 Conclusions: MeDUsA rapidly and accurately quantifies axons in Drosophila eye. By

41 simplifying and automating time-consuming manual efforts requiring significant

42 expertise, it enables large-scale, complex research efforts on axonal degeneration, such 43 as screening to identify genes or drugs that mediate axonal toxicity caused by ND disease 44 proteins.

\section{Keywords:}

47 Axonal degeneration, Quantification, Standalone program, Drosophila,

$48 \quad$ Neurodegenerative disease 


\section{Background:}

51 Neurodegenerative diseases (NDs) are disorders in which certain groups of neurons in

52 the brain and spinal cord involved in cognitive and motor function are gradually lost.

53 Molecular genetic studies have identified causative genes and risk factors and elucidated

54 the mechanisms of pathogenesis at the molecular level. These findings revealed that

55 structural defects and aggregation of disease-associated proteins underlie

56 neurodegenerative processes [1]. It has become available to examine the effects of novel

57 mutations found in human diseases like NDs, whether they result in the loss of gene

58 function or gain of toxic function, using various model organisms. Among them,

59 Drosophila has various advantages as a neuronal disease model. For example, 1) gene

60 function can be analysed without strongly considering the compensation of gene function

61 by duplication since there are relatively few duplicated genes in the genome; 2) research

62 sample sizes can be large because individuals are small, inexpensive and easy to breed;

63 3) the short life cycle allows rapid genetic analysis and 4) the organism has a compact

64 brain, making it possible to analyse at the level of neural circuitry and behaviour necessary for higher functions such as learning, memory and sleep. Taking advantage of these features, Drosophila is widely used in studies on human diseases. Further, it has

67 been reported that the expression of a human disease-associated protein in Drosophila

68 induces toxicity even in flies [2-6], demonstrating the conservation of molecular 69 mechanisms between humans and flies.

Trinucleotide repeat disorders are human diseases caused by the expansion of

71 CAG repeats in the protein-coding regions of causative genes. Spinocerebellar ataxia type

723 (SCA3), also known as Machado-Joseph disease, is a neurodegenerative disease caused 
73 by repeated elongation of glutamine. In Drosophila, the expression of these extended

74 polyglutamine repeats not only formed inclusion bodies similar to those in humans, but 75 also caused degeneration [2]. CAG repeats are also found in Huntingtin (HTT), the gene 76 responsible for Huntington's disease, which is another autosomal dominant 77 neurodegenerative disease. In experiments in which polyglutamine-extended HTT was expressed in the photoreceptors of Drosophila, inclusion bodies formed and the polyglutamine-extended HTT induced neurodegeneration [3]. Additionally, in Parkinson's disease (PD), a neurodegenerative disorder characterised by the loss of 81 dopaminergic neurons in the substantia nigra, formation of Lewy bodies and impaired 82 motility, expression of the causative gene synuclein alpha (SNCA) in Drosophila caused the loss of dopaminergic neurons, the formation of fibrous intraneuronal inclusions

84 containing Alpha-synuclein ( $\alpha$ Syn) and motor dysfunction [4]. Furthermore, the expression of the microtubule-associated protein Tau, which is involved in Alzheimer's disease, in all neurons of Drosophila led to the observation of progressive

87 neurodegeneration [5]. Thus, Drosophila models expressing human disease-causing genes can reproduce the characteristics of human diseases, thereby enabling powerful genetic approaches to study various NDs such as polyglutamine disease, synucleinopathy and tauopathy. Using these ND models, large genetic screens can be performed to explore

91 unknown protein networks in which disease-causing proteins interact. The homologues

92 of the candidate network members can then be identified in the human genome to 93 determine whether they are susceptibility genes of the disease of interest. In one example 94 of a disease study of amyotrophic lateral sclerosis (ALS), genetic screening using this experimental paradigm identified that ATXN2 is involved in ALS pathogenesis 
97 TDP-43, which is a DNA/RNA-binding protein implicated in several NDs. The discovery

98 of ATXN2 accumulation in the spinal cord of human ALS is but one example revealed using this screening approach in Drosophila.

The rough eye phenotype (REP), which is frequently used to investigate genetic

101 interactions in Drosophila, is also used in disease research as a convenient and quick way to assess the toxicity of ectopically expressed genes. The phenotypic assay uses the Gal4/UAS method [8] to evaluate the toxicity of a gene of interest by expressing it specifically in the eye using the eye-specific Gal4, GMR-Gal4 and observing eye structure. In fact, this fly eye assay has identified several modifiers that inhibit or enhance the toxicity caused by pathogenic factors such as Tau [9-11], $\alpha$ Syn [12-15], TDP-43

$107[16,17]$ and polyglutamated HTT [18-20]. Moreover, drug screenings can be performed by adding compounds to fly food, and compounds that reduce disease toxicity can be identified [21,22]. Although REP has been evaluated qualitatively in most previous

110 studies, several research groups have recently reported methods for the quantitative

111 evaluation of REP [23,24]. However, it remains unclear whether REP reflects

112 neurodegeneration completely as the formation and geometric defects of cell clusters are

113 assessed only within the ommatidium, which contains cone and pigment cells. To assess

114 neurodegeneration more fully in Drosophila, several other parameters related to 115 neurodegeneration, as measured by protein aggregation number, vacuolar size and 116 number and retinal thickness, have been previously developed [25]. However, these 117 systems also indirectly observe neurodegeneration, and therefore, a direct quantitative 118 method for accurately measuring neurodegeneration is required.

119 Axonal degeneration is a representative pathology of neurodegeneration, and 120 the magnitude of neurodegeneration can be accurately evaluated by its quantification. To 
121 date, there are few methods for quantifying axonal degeneration [26,27]. Further, they

122 are time-consuming, requiring the manual quantification of axonal number or depend on

123 the expertise of experimenters to accurately classify the degree of degeneration. However,

124 biological images contain noise, and variable signal intensities are often observed among

125 samples. Additionally, the three-dimensional (3D) structure and angle are never uniform

126 between images. Thus, to recognise the semantic region from diverse image data, the eye

127 of a trained researcher can flexibly and accurately extract specific phenomena to be

128 analysed, but such time-consuming methods are not suitable for screening to identify

129 genes or compounds that modify the pathology and requires quantifying large numbers

130 of samples. Therefore, robust image processing systems other than the human eye are

131 required to evaluate axonal degeneration simply and quickly. In recent years, image

132 processing technology has made remarkable progress, especially the development of

133 deep-learning technology using convolutional neural networks (CNNs), which has

134 greatly advanced the field of image recognition, and is also true in the field of biological

135 images [28]. In addition, although conventional image processing can extract signal

136 regions, it is difficult to extract semantic regions, but CNN has demonstrated high

137 performance in semantic domain segmentation. In particular, U-Net [29] is an

138 architecture of CNNs designed for biological image analysis, and U-Net and its derived

139 architectures have been used for segmentation tasks in the area of biological image

140 analysis with great success [30-33].

141 In an accompanying study, we developed a novel method to directly quantify

142 axonal degeneration using R7, a photoreceptor neuron type in Drosophila, as a model

143 [34]. In this method, axon terminals are manually excised from the confocal microscope

144 z-stack image series, and degeneration is quantified by manual counting the number of 
145 axon terminals. This allowed quantifying even minor axon losses; therefore, even very

146 early stages of axonal degeneration phenomena. Nonetheless, its throughput was not

147 sufficient for larger scale screens. To automate the method, we established here a novel

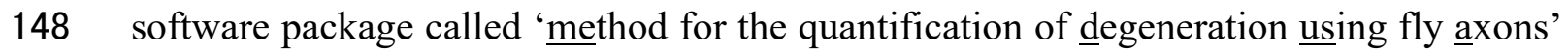

149 (MeDUsA) by combining deep learning with a Python-based counting system. Using this

150 software, we assessed the effects on axons among the causative genes of several NDs and

151 found that they exhibited axonal degeneration. Additionally, no significant correlation

152 was detected between the number of axons and the REP. MeDUsA provides direct and

153 rapid quantitation of axonal degeneration in Drosophila, making it a powerful tool that

154 can be used in disease and developmental studies.

156 Results:

157 Rough eye phenotype is insufficient to speculate on gene effects in axonal

158 degeneration

159 The Rough eye phenotype (REP) assay has been extensively used to study 160 neurodegenerations (NDs) [35]; however, there is uncertainty whether REP accurately

161 reflects axonal degeneration. We performed a modifier genetic screening designed to

162 identify genes that modulate the toxicity of TDP-43 by observing retinal and axonal 163 degeneration phenotypes. The Drosophila photoreceptors R7 and R8 project their axons

164 directly from the compound eye retina through the primary optic ganglion lamina to the 165 secondary optic ganglion medulla (Fig. 1A). For this screening, a fly line with eyespecific expression of TDP-43 ${ }^{\mathrm{G} 298 \mathrm{~S}}$, which is an ALS-associated mutation of TDP-43 [36],

167 using GMR-Gal4 [37,38] was generated. These flies were crossed with 99 candidate RNA 168 interference (RNAi) lines (Fig. 1B; see Materials and Methods). In the first screening 
round, we observed the eye phenotype because the eye-specific expression of TDP$43^{\mathrm{G} 298 \mathrm{~S}}$ causes REP as previously reported [39]. As a result, 14 of 99 RNAi lines were

171 identified that suppress REPs (Fig. 1B, 1C). Next, we evaluated the axonal degeneration

172 of R8 retinal axons, as the second screening round (Fig. 1B, 1C). To quantify the axonal

173 degeneration, we calculated the ratio of degenerated axons in the part of the optic lobe

174 that is easy to observe each axon. We classified an axon as degenerated when the axon

175 was fragmented. Eye-specific expression of TDP-43 displayed axonal degeneration of R8

176 axons one day after eclosion (Fig. 1C). We expected that REPs identified in the first

177 screening round would be consistent with the morphology of the R8 axons under the

178 assumption that RNAi lines which suppressed REPs rescued the axonal degeneration.

179 Surprisingly, the REP results did not always match those of axonal degeneration.

180 Knockdown of $D s k$, a neuropeptide-encoding gene identified in only crustaceans and

181 insects, in the background of TDP-43 expression strongly suppressed REP; however, Dsk

182 knockdown significantly promoted axonal degeneration (Fig. 1C; quantified in 1D). In

183 addition, the knockdown of 10 genes did not affect axonal degeneration, whereas 3 genes

184 (mle, faf and caz) rescued the degeneration (Fig. 1C; quantified in 1D). Thus, although

185 the REP assay is a simple and powerful method for assessing toxicity, it is insufficient to

186 evaluate axonal degeneration. Further, the quantitative method we used to assess

187 degeneration in this screening was also insufficient for precise quantification because it

188 was a subjective determination whether an axon was degenerated (i.e. fragmented) or

189 intact. Other limitations include the fact that axons which are completely lost cannot be

190 counted and all R8 axons were not counted from the dorsal view due to limits of the

191 confocal microscope scanning time and depth at which the sample could be viewed

192 cleanly. Additionally, depending on the person performing the method and region being 
quantified, the results would display a high degree of variability. Therefore, we developed an automatic system, MeDUsA for the unbiased quantitative evaluation of axonal degeneration in Drosophila.

197 The process flow for using MeDUsA

198 To quantify R7 axons using MeDUsA, the first step is to prepare samples of the 199 Drosophila brain (Fig. 2A and 2B). Dissection and immunostaining were performed as 200 previously described $[34,40]$.

In the second step, axon termini were identified and quantified. For this purpose,

202 we developed MeDUsA to enable non-experts to accurately evaluate axonal degeneration and to save time. MeDUsA utilises a combination of tools available in Python that allows

204 the task of masking axons to be performed by pre-trained deep learning, followed by automatic counting of axon terminals after masking. This software enables researchers to quantify the number of R7 axons readily and quickly, taking 50 seconds per sample on a general desktop workstation (CPU: Intel Core i7 9800X 3.8 GHz, RAM: DDR4 128 GB).

210 infer it from the axon terminal signals in the sparsity, and there are areas where it is

211 difficult to determine the surface area with a single z-slice. Therefore, we trained the 2D-

212 U-Net to generate a surface mask of three slices by inputting three slices as three channels,

213 including the slice before and after the slice of interest. Then, during inference, only the

214 mask of the centre slice was used. The training and testing datasets included both normal 215 and abnormal images. A total of 16,114 images in 199 samples were used for training and 
2161,375 images in 16 samples were used for testing. By using three channels, the dice score

217 improved from 0.815 to 0.847 compared to using a single channel.

218 After mask generation and axon terminal extraction, the number of axonal

219 terminals was counted automatically (Fig. 2D). To do this, we first filtered the regional

220 maxima to remove background, followed by binarisation using adaptive thresholding.

221 The surface area was then extracted using mask images. We performed a Euclidean

222 distance transformation to obtain peaks, which were used as seeds to perform 3D

223 watershed to obtain each axon terminal candidate. Finally, candidates below 20 voxels

224 (equivalent to a radius of about $1.68 \mu \mathrm{m}$ ) were eliminated, and the remaining ones were

225 counted as axon terminals. This process was fully automated and allowed us to stably detect axon terminals without adjusting parameters for each sample.

227

228 Ectopic expression of causative genes of NDs causes axonal degeneration in R7

229 neurons

230 Using our MeDUsA, we evaluated the effect of mutations in proteins responsible for NDs on axonal degeneration in Drosophila. We expressed either wild-type or well-known mutant alleles of human causative genes for neurodegenerative diseases ( $\alpha$ Syn, Tau, TDP-43 and HTT) in photoreceptor axons using GMR-Gal4 and predicted the number of

234 axonal terminals in 1-day-old adults. The ectopic expression of wild-type $\alpha$ Syn, which is

235 a causative gene of PD [41], in photoreceptor axons did not show a significant reduction in axonal number compared to control, whereas the expression of A53T-mutated $\alpha$ Syn, a

237 well-known pathogenic allele associated with familial PD [42], caused a significant 238 reduction in the number of axons compared to control (Fig. 3A-C; quantified in 3K). 239 However, there was no significance between wild-type and A53T-mutated aSyn. Next, 
240 we found that expression of wild-type Tau (Tau $\left.{ }^{\mathrm{WT}}\right)$, which is implicated in Alzheimer's

241 disease, caused significant axonal degeneration, and the expression of Tau with the

242 R406W mutation, which is a missense mutation identified in families diagnosed with

243 frontotemporal dementia and parkinsonism linked to chromosome 17 [43], enhanced the

244 degeneration (Fig. 3D, 3E; quantified in 3K). Interestingly, the expression of Tau with

245 the S2A mutation, a mutation with impaired phosphorylation capabilities, significantly

246 suppressed axonal degeneration compared to $\mathrm{Tau}^{\mathrm{WT}}$ (Fig. 3F), indicating that

247 phosphorylated Tau exhibits toxicity. Next, we evaluated the expression of both wild-

248 type and A315T-mutated [44] TDP-43, which has been identified as the major disease

249 protein in ALS, in photoreceptor axons, and found that both displayed axonal

250 degeneration, although degeneration was milder in the A315T mutant than in wild-type

251 TDP-43 (Fig. 3G, 3H; quantified in 3K). Finally, we found that ectopic expression of

252 wild-type HTT $\left(\mathrm{HTT}^{\mathrm{Q} 0}\right)$, the causative gene of Huntington's disease, did not cause axonal

253 degeneration, whereas the expression of HTT with a pathogenic polyQ tract of 128

254 repeats $\left(\mathrm{HTT}^{\mathrm{Q}}{ }^{28}\right.$ ) significantly reduced the number of R7 axons (Fig. 3I, 3J; quantified

255 in 3K). These results show that the ectopic expression of causative genes of human NDs

256 induced axonal degeneration in Drosophila, and well-known pathogenic mutations

257 enhanced degeneration, except for TDP-43. Our findings demonstrate that the toxicity of

258 various human disease causative proteins can be reliably assessed across species using

259 our quantitative axonal degeneration fly model.

To evaluate the performance of MeDUsA, we quantified the axonal number

261 of the same sample set manually (Fig. 4A). This manual method enabled us to carry out

262 precise quantification of axonal number; however, manual quantification is time-

263 consuming and several parameters have to be adjusted for each sample to detect axon 
terminals. The MeDUsA measurements were lower than the manual measurements (Fig. 4B). This is due to the severity of the setting (Fig. 2D) for recognising the axon terminal in automated quantification. If the setting is further set loose, the false positive count

267 increases. At present, this is the limitation of the MeDUsA. Nevertheless, the system

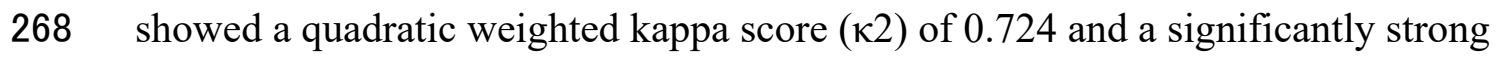
positive correlation with manual counting (individual values, $R^{2}=0.887, p<0.001$, Fig. 4C; mean value per genotype, $R^{2}=0.944, p<0.001$, Fig. 4D). Taken together, these

271 findings demonstrate that MeDUsA automatically and rapidly counts axonal number in 272 a preparation accessible for genetic screening.

\section{REP does not match the axonal degeneration phenotype}

275 Next, we evaluated the correlation between axonal degeneration phenotype and the REP.

276 To quantify REP severity, we used Flynotyper that calculates the phenotypic score from

277 the disarray of the ommatidia [24]. We expressed the same set of causative genes for NDs

278 in the eye using GMR-Gal4 as in axonal degeneration experiments and quantified the 279 degree of phenotypic severity of eye phenotypes in 1-day-old adults. We found that the expression of either the wild-type or pathogenic allele of aSyn did not significantly

281 increase the phenotypic score compared to control (Fig. 5A-C; quantified in 5K),

282 although the expression of the pathogenic alleles of $\alpha$ Syn caused axonal degeneration

283 (Fig. 3K). In contrast, REP severity was consistent with the reduction of axonal number when the wild-type and mutant alleles of Tau were expressed (Fig. 5D-F; quantified in 5K). Wild-type and R406W-mutated Tau, both of which caused axonal degeneration, showed a significantly increased phenotypic score, whereas the S2A mutation did not 
we found that TDP-43 expression showed an obvious discrepancy between the two phenotypes. As described earlier, wild-type TDP-43 is more toxic than A315T-mutated TDP-43 on axonal degeneration (Fig. 3K). However, the A315T mutation caused a

291 significant increase in the REP score compared with wild-type TDP-43 (Fig. 5G, 5H;

292 quantified in 5K). HTT expression also exhibited differences between axon and eye phenotypes. In contrast to axonal degeneration phenotypes, the ectopic expression of HTT $^{\text {128 }}$ did not cause REPs (Fig. 5I, 5J; quantified in 5K). Finally, we determined the correlation between the average axonal number and average phenotypic score of rough eye in each genotype, and found a negative correlation, but the correlation was not statistically significant $(R=-0.467, p=0.174)$. Taken together, our findings show that REP scoring is not exactly consistent with the degree of axonal degeneration.

\section{Discussion:}

301 We recently developed a method that precisely quantifies the axonal number of retinal

302 R7 neurons by creating a mask which extracts the axon terminals [34]. In the present study, we developed and tested MeDUsA, a software that automatically creates masks and counts axon terminals using a combination of deep learning and Python (Fig. 2). By using this software, the number of R7 axons can be automatically quantified easily and quickly from confocal images. Conventionally, REP is frequently used to assess

307 neurotoxicity in fly studies because it does not require special equipment and can be readily performed; however, we found that the severity of REP is not always consistent axonal degeneration of R7 are not accompanied by cell death [34]. Therefore, we propose

311 that ND research can be conducted more efficiently by combining REP with our method 
312 when the focus is axonal degeneration. Although several models for evaluating axonal

313 degeneration in Drosophila have been reported [26,27,34], they are technically difficult

314 and exceedingly time-consuming to evaluate axonal degeneration in large-scale

315 experiments such as screening as the evaluation of degeneration in these models is

316 subjective or require manual measurement. However, by using MeDUsA, it is not only

317 possible to identify factors and chemical compounds that inhibit axonal degeneration in

318 the fly model of ND by screening but also to easily evaluate axonal toxicity of new or

319 undiagnosed variants of pathological proteins of ND.

320 According to our modifier screening using REP to identify genes that suppress

321 TDP-43 ${ }^{\mathrm{G} 298 \mathrm{~S}}$ toxicity, we identified 14 candidate genes. Further investigation focused on

322 axonal degeneration showed that the knockdown of three genes (mle, caz and faf)

323 suppressed toxicity, whereas Dsk knockdown promoted it (Fig. 1C, 1D). Dsk encodes the

324 cholecystokinin-like neuropeptide Drosulfakinin and has been reported to be involved in

325 feeding behaviour, aggression and larval avoidance [45-48]. A previous study reported

326 that Dsk is also involved in synaptogenesis in the neuromuscular junction in cooperation

327 with a putative cholecystokinin-like receptor [49]. Studies in mouse models have shown

328 that the pathogenic form of TDP-43 has harmful effects on synapses. For example,

329 hyperexcitability has been observed [50] and spine density has been reduced [51].

330 Therefore, our results suggest that Dsk reduction causes synapse dysfunction and make

331 axons fragile, whereby axonotoxicity of TDP-43 ${ }^{\mathrm{G} 298 \mathrm{~S}}$ is enhanced. Named by its male-

332 specific lethal phenotype in loss-of-function mutants, mle (maleless) encodes an RNA

333 helicase and is a member of the Male-Specific-Lethal transcription complex, which is

334 involved in dosage compensation in males [52]. The homologue of $m l e$ in human, $D H X 9$,

335 was reported to encode a TDP-43-interacting protein [53]. Furthermore, a previous study 
reported in a fly model that knockdown of mle exacerbated neurodegeneration caused by

337 the expression of expanded UGGAA, which is considered responsible for spinocerebellar

338 ataxia type 31 (SCA31), whereas overexpression of wild-type TDP-43 suppressed

339 expanded UGGAA-induced toxicity [39]. Thus, mle may be involved in the RNA-

340 dependent toxicity of TDP-43. caz (cabeza), which encodes a RNA-binding protein, is a

341 fly homologue of FUS/TLS, which is another major causative gene for ALS. Physical

342 interactions between human FUS/TLS and TDP-43 have been reported in mammalian

343 cultured cells $[54,55]$, and it has been suggested that FUS/TLS is genetically located

344 downstream of TDP-43 in fly and fish models [56,57]. Therefore, the attenuation of

345 axonal degeneration by caz knockdown may be due to the suppression of excess unknown

346 downstream factors of TDP-43 or caz itself or both. The influence of $f a f$ (fat facets),

347 which encodes a deubiquitylated enzyme and is a fly homologue of USP9X, on

348 neurodegeneration varies among fly ND models. Lee et al. reported that FAF enhanced

349 the toxicity of amyloid precursor protein (APP) and A $\beta-42$. The co-expression of FAF

350 with either APP or A $\beta-42$ enhanced REP and knockdown of faf suppressed the post-

351 synaptic toxicity of APP and A $\beta-42$ [58]. Another group revealed that reduced levels of

$352 f a f$ enhanced retinal toxicity of HTT [19]. As FAF deubiquitinates different substrates in

353 these diseases, it may be reflected in the different effects on the toxicity of causative

354 proteins of the ND. Further analysis of these genes would shed light on the molecular

355 mechanisms underlying axonotoxicity by TDP-43 ${ }^{\mathrm{G} 298 \mathrm{~S}}$.

Using the automatic quantification method developed in this study, we 
studies have directly visualised and quantitatively evaluated axonal degeneration such as

361 that demonstrated in this study using MeDUsA. Our findings show that MeDUsA is highly extensible and can be used in different NDs to demonstrate how a causative gene affects axonal degeneration. cell body and leads to neuronal death $[60,61]$. The A53T mutation is a well-known toxic mutation of $\alpha$ Syn that aggregates more rapidly and forms fibrils than wild-type, and many studies using animal models and induced pluripotent stem (iPS) cells derived from

371 patients with PD have reported high neurotoxicity [62-64]. In our study, a significant

372 reduction in the number of axons was observed between control and $\alpha \mathrm{Syn}^{\mathrm{A} 53 \mathrm{~T}}$-expressed

373 flies, but no statistically significant difference was observed between wild-type and A53T

374 (Fig. 3K). A possible reason for the non-significant difference is that quantification was performed too early (1-day-old adult), and differences between the wild-type and A53T mutation may be observed if quantified several weeks after eclosion. Tau is a microtubule-associated protein that binds to microtubules and

378 maintains their stability in neurons. In tauopathies, Tau is thought to be dissociated from 379 microtubules by excessive phosphorylation and aggregate, causing a dying-back pattern 380 of neurodegeneration [65]. Although several studies have reported increased toxicity with 381 the R406W mutation, a change in the phosphorylation status remains controversial.

382 Studies using in vitro and patient-derived iPS cells have reported that R406W mutant tau 383 is less phosphorylated than wild-type tau, whereas excessive phosphorylation has been 
384 observed in both murine model and post-mortem patient brain [66-69]. Although

385 previous studies reported that overexpression of $\mathrm{Tau}^{\mathrm{R} 406 \mathrm{~W}}$, which was used in this study, 386 displayed high toxicity in REP compared to Tau ${ }^{\mathrm{WT}}$ in Drosophila [5,70], it may be due 387 to positional effects of the UAS insertion site [71]. Consistent with previous reports, we 388 found that $\mathrm{Tau}^{\mathrm{R} 406 \mathrm{~W}}$ overexpression exerted a more toxic effect on axons than $\mathrm{Tau}^{\mathrm{WT}}$ 389 overexpression (Fig. 3K). component of ubiquitinated aggregation in the neurons of patients with ALS and

392 frontotemporal lobar degeneration (FTLD) [72]. Numerous studies that have investigated 393 the physiological function of TDP-43 have revealed that TDP-43 is involved in various aspects of RNA metabolism, and these disturbances may be responsible for the pathogenesis of ALS and FTLD [73]. In many model organisms, both overexpression and loss-of-function of TDP-43 result in reduced longevity and motor function [74-77]. The

397 overexpression of wild-type TDP-43 in Drosophila mushroom body neurons of the 398 olfactory memory centre causes axonal degeneration [78], whereas the effects of the 399 A315T mutation on TDP-43 toxicity are controversial in Drosophila. Guo et al. reported 400 that TDP-43 ${ }^{\mathrm{A} 315 \mathrm{~T}}$ was more toxic to motor neurons than TDP-43 ${ }^{\mathrm{WT}}$, whereas Patricia et 401 al. reported that TDP-43 ${ }^{\mathrm{WT}}$ showed severe toxicity compared to TDP-43 ${ }^{\mathrm{A} 315 \mathrm{~T}}$, except for 402 larval locomotor activity $[79,80]$. In our present result of retinal R7 axons, we found that 403 wild-type TDP-43 showed higher axonal toxicity than the A315T mutation (Fig. 3K). 404 Although TDP-43 is primarily expressed in the nucleus, the aggregation of TDP-43 in the cytoplasm causes toxicity. As wild-type TDP-43 may be increased in the cytoplasm if it

406 is overexpressed, it is important to realise that differences in expression levels in each 
407 experimental system may contribute to differences in the respective results. TDP-43 may

408 also have different toxic effects on different neuronal cell types.

In Huntington's disease, CAG repeat expansion of HTT produces abnormal

410 RNA and protein, leading to neuronal dysfunction and eventual cell death. The normal

411 allele of HTT contains fewer than 26 CAG repeats, whereas 36 repeats or more are

412 associated with Huntington's disease [81]. A study using a mouse model of HD and

413 human patients indicated that the degeneration of the callosal axon was seen before

414 symptoms were observed, suggesting that the mutant HTT caused dying-back

415 neurodegeneration [82]. Furthermore, CAG repeat number-dependent cytotoxicity has

416 been reported in Drosophila [83]. In this study, as in previous reports, $\mathrm{HTT}^{\mathrm{Q} 128}$ showed 417 axonal toxicity but $\mathrm{HTT}^{\mathrm{Q} 0} \operatorname{did}$ not (Fig. 3K).

418 Axonal degeneration is observed not only in NDs but also in Wallerian 419 degeneration (WD), which is the axotomy-induced distal degeneration of an axon, 420 causing a decline in neuronal function. The mechanisms of axonal degeneration in NDs 421 and WD are partly overlapping but not identical. Wlds is a fusion protein that slows WD; 422 it confers a protective effect from degeneration in animal models of progressive motor 423 neuropathy and PD, but does not ameliorate in ALS model [84-86]. These findings 424 suggest that each pathological protein causes axonal degeneration by a different 425 mechanism, but the detailed molecular mechanisms are still poorly understood. However, 426 by using our method, it is anticipated that research focusing on axon degeneration will be 427 facilitated, thereby enabling the elucidation of the pathological mechanism of axonal 428 degeneration by the causative genes of ND and WD. In turn, a better understanding of the 429 underlying molecular mechanisms of axonal degeneration is promising for developing 430 therapies that inhibit or delay the onset of ageing, NDs and WD. 
432 extracts axon terminals from a confocal z-stack image. The image processing capabilities

433 afforded by machine learning are powerful, and recently, many quantitative and

434 segmentation methods using machine learning have been published [87-90]. MeDUsA is

435 a Python-based method specifically designed to count axons; however, it only quantifies

436 the presence of axons and does not capture pre-degenerative signs such as swelling or

437 fragmentation of axon terminals. Such changes are currently more precisely captured only

438 with a manual method [34]. An important future step will be to extend MeDUsA to

439 perform a more comprehensive quantitative analysis of morphological and cell biological

440 properties, such as the size and shape of axonal termini, the number or organisation of

441 pre-synapses, of mitochondria or other organelles in axonal terminals, to enable more

442 detailed studies of pathological mechanisms of ND.

443

\section{Conclusions:}

445 In this study, we developed MeDUsA for automatically quantifying the number of axons

446 in retinal R7 neurons in Drosophila with high reliability and robustness. It combines pre-

447 trained deep-learning models with a Python-based quantification system. Using our easy-

448 to-use software, we demonstrated the causative proteins of NDs actually caused the

449 axonal degeneration. We also confirmed that the severity of REP and axonal number were

450 not significantly correlated. MeDUsA is a valuable tool for the unbiased and rapid

451 quantification of axonal degeneration in genetic or pharmacological modifier screening

452 using Drosophila as a model.

\section{$454 \quad$ Materials and Methods:}




\section{Fly Strains}

456 Flies were maintained at $25^{\circ} \mathrm{C}$ on standard fly food. Female flies were used in all

457 experiments except for those shown in Figure 1 to adjust the number of retinal axons.

458 40D-UAS (VDRC ID 60101) was obtained from the Vienna Drosophila Resource Center

459 (VDRC) in Vienna, Austria. GMR-Gal4 (III) (\#8121), Rh6-Gal4 (\#7459), Brp-FSF-GFP

460 (\#55753), UAS-myr-RFP (\#7119), UAS-SNCA (\#8146), UAS-SNCA ${ }^{A 53 T}$ (\#8148), UAS-

Tau $^{\text {S2A }}$ (\#51364), tub-Gal80 ${ }^{T S}$ (\#7017 and \#7019), UAS-marf RNAi (\#55189), UAS-opa1

(\#64315) and 99 strains for RNAi screening were obtained from the Bloomington

Drosophila Stock Center (BDSC, Bloomington, IN, USA). Details are provided in Sup.

Table 1. GMR-Gal4(II) [37,38] was used for the expression of transgenes in the

Chi-Hon Lee [92], and Sens-flippase [93] was kindly provided by Dr. S. L. Zipursky.

$U A S-T a u[5]$ and $U A S-T_{a u}^{R 406 W}$ [5] were gifted by Dr. M. B. Feany. UAS-HTT ${ }^{Q 0}$ [94]and

$U A S-H T T^{Q 128}$ [94] were graciously given by the Dr. J. T. Littleton. UAS-TDP-43(strong),

$U A S-T D P-43^{A 315 T}$, and $U A S-T D P 43^{G 298 S}$ were described previously $[39,95]$.

\section{Immunohistochemistry and imaging}

473 Immunohistochemistry and sample preparation were performed as described previously

$474[34,40]$. The following antibodies were used: mouse anti-Chaoptin $(24 \mathrm{~B} 10,1: 25$;

475 Developmental Studies Hybridoma Bank, Iowa City, IA, USA), anti-mouse Alexa Fluor

476488 (1:400; Thermo Fisher Scientific) and anti-mouse Alexa Fluor 568 (1:400; Thermo

477 Fisher Scientific). The insect pins were used because they are $0.1 \mathrm{~mm}$ in diameter and fit

478 the thickness of a fly brain. A coverslip was added and slides were mounted using 
479 Vectashield mounting medium (Vector Laboratories). Images were captured using a

480 FV3000 confocal microscope (Olympus, Tokyo, Japan). We then obtained brain images

481 using a confocal microscope set to a $60 \times$ immersion objective (1.4 numerical aperture)

482 and a $1 \times$ digital zoom. During the scan, we set the step size to $1 \mu \mathrm{m}$ and generated $60-90$

483 optical sections of the second optic ganglion medulla, including all R7 axon terminals.

484 They are for the images shown in Figure 2, 3 and 6. In Figure 1, samples were scanned

485 using an A1 confocal microscope (Nikon, Tokyo, Japan). Images were processed using

486 either IMARIS 9.6.0 (Bitplane) or Fiji software, which is an open-source image analysis

487 software [96].

488

489

Eye imaging using bright-field microscopy and quantification of morphological eye

490

defects

491 For light microscopy imaging of adult eyes, 1- to 3-day-old flies, which were reared at $49229^{\circ} \mathrm{C}$ for the expression of $\alpha \mathrm{Syn}$, Tau and HTT and at $25^{\circ} \mathrm{C}$ for the expression of TDP49343 , were immobilised by freezing at $-20^{\circ} \mathrm{C}$, and separated fly heads were mounted on 494 labelling tape (Shamrock Labels, IL, USA). The flies were then imaged using an OM-D 495 E-M5 digital camera (Olympus) and SZX16 microscope with $6.3 \times$ magnification 496 (Olympus). About 20 photographs were taken with the focus shifted slightly from the 497 centre of the eye to the edges. Each slice was depth-synthesised using Photoshop CC 2017 498 (Adobe). The edges of the eyes were then trimmed and processed using Flynotyper 1.0 to 499 quantitatively assess morphological defects in Drosophila eye [24].

500

501 Combined rough eye and axonal degeneration phenotype screening 
502 First, candidate genes for screening were selected as the phenotype in the QuickSearch

503 of FlyBase (https://flybase.org/) and searched for 'synapse' in the 'Tissue/cell affected'

504 category. The 401 genes hit using this method were further narrowed down using settings

505 of 'Higher' and 'Moderately High' with 'Adult Head' in the modENCODE Expression

506 Data of FlyBase, and 99 genes available for RNAi lines at the BDSC were selected as 507 candidate genes.

508 For the initial screening, we searched to identify a factor that suppressed REP

509 to serve as an indicator of neurodegeneration. In this study, we used a transgene of TDP-

$51043^{\mathrm{G} 298 \mathrm{~S}}$ expressed by the eye-specific GMR-Gal4 driver to induce REP. We then

511 evaluated each RNAi line of the candidate genes, and the genes in which REP was

512 suppressed were selected. We then imaged the compound eyes of Drosophila using an

513 EOS Kiss X4 digital camera (Canon, Tokyo, Japan) and MZ FLIII fluorescence stereo

514 microscope (Leica, Wetzlar, Germany).

515 Next, as the second screening, R8 axons were visualised to further narrow the

516 candidate factors based on the ability to recover from axonal degeneration. To achieve

517 this, myrRFP was expressed using the Rh6-Gal4 driver. The genotype was Sens-

518 flippase/+; Rh6-Gal4/UAS-TDP-43 $3^{G 298 S ; \quad B r p-F S F-G F P, ~ U A S-m y r-R F P / U A S-R N A i . ~}$

519 Knockdown was performed on 15 candidate genes that suppressed the REP, and the axons

520 were observed using an A1 confocal microscope (Nikon).

521

522 Training set generation

523 To quantify both normal and degenerated axons with sufficient accuracy, we used

524 samples with phenotypes of axonal degeneration induced by either light stimulation or

525 mitochondrial dysfunction for machine learning. 
527 that constant light stimulation caused progressive axonal degeneration [34]. Taking 528 advantage of this phenomenon, we used samples of various time points under constant

529 light from normal states to severe axonal degeneration for machine learning. In detail, 530 experimental samples at $1(n=16), 3(n=16), 5(n=20), 7(n=16), 9(n=14), 11(n=$ 531 14) and 13 days $(n=13)$ under constant light were used. Samples $(n=10$ and $n=19)$ 532 were used on day 1 and day 13 in a 12-h light/dark cycle as controls, respectively. The genotype was GMR-w-RNAi/w-; lexAop-syb-spGFP1-10, UAS-CD4-spGFP11/Rh4-

534 LexA; ortC2b-Gal4/+.

535 For machine learning, we also induced photoreceptor axonal degeneration by 536 knocking down Marf or Opa1, both of which are required for mitochondrial fusion. The genotypes were GMR-Gal4/40D-UAS; tub-Gal80 ${ }^{\text {ts }} /+(\mathrm{n}=6)$, GMR-Gal4/UAS-marf RNAi; tub-Gal80ts/+ $(\mathrm{n}=9)$ and GMR-Gal4/tub-Gal80 ${ }^{t s} ; U A S-o p a 1$ RNAi/+ $(\mathrm{n}=20)$.

539 The flies were reared in a permissive temperature $\left(20^{\circ} \mathrm{C}\right)$, and after eclosion, knockdown

540 was induced by rearing the flies in a restrictive temperature $\left(29^{\circ} \mathrm{C}\right)$ and were dissected 4

541 weeks later. Samples reared under control conditions at $20^{\circ} \mathrm{C}$ for 4 weeks after eclosion

542 were also used for machine learning. The genotypes were GMR-Gal4/40D-UAS; tub-

543 Gal80 $0^{t s} /+(\mathrm{n}=14)$, GMR-Gal4/UAS-marf RNAi; tub-Gal80 $0^{t s} /+(\mathrm{n}=20)$, and GMR-

544 Gal4/tub-Gal80's; UAS-opal RNAi/+ $(\mathrm{n}=10)$. created a mask covering the axonal terminal and used the scanning data and the mask as

547 a training set. 
550 A variant of 2D-U-Net was used for surface mask prediction. The original images were

$551 \mathrm{~N} \times 512 \times 512$ in size for each sample, and for each $\mathrm{z}$-slice, the image before and after it

$552(\mathrm{z}-1, \mathrm{z}$ and $\mathrm{z}+1)$ were combined to form the $\mathrm{N} \times 512 \times 512 \times 3$ size. As the terminal

553 slice does not have $\mathrm{z}-1$ or $\mathrm{z}+1$, a blank image was used instead. For training, we used

554 mask images of size $\mathrm{N} \times 512 \times 512 \times 3$, and for inference, we obtained mask prediction

555 images of size $\mathrm{N} \times 512 \times 512$ by discarding the channels corresponding to each $\mathrm{z}-1$

556 and $\mathrm{z}+1$ from the $\mathrm{N} \times 512 \times 512 \times 3$ output and retaining only $\mathrm{z}$. As post-processing,

557 opening and closing operations were used to exclude small blobs and to fill in holes. Then,

558 from the original image, only the area corresponding to the obtained surface mask image

559 was extracted. To detect the axon terminal, the background image was first created by

560 morphological reconstruction and then filtered regional maxima by subtracting it from

561 the original image to remove the background. Next, the image was binarised by adaptive

562 thresholding, and 3D watershed was performed using the peak obtained by calculating

563 the Euclidean distance as a seed. Finally, blobs below 20 voxels were excluded. These

564 processes were calculated using Python (v3.6.7), NumPy (v1.17.3), TensorFlow (v1.13.2),

565 scikit-image (v0.16.2), SciPy (v1.3.2) and OpenCV (v4.2.0).

567 Experimental design and statistical analyses

568 Experimental analyses were performed using Prism 8 (GraphPad Software, San Diego,

569 CA, USA). All quantifications were performed by experimenters who were blind to the

570 genotype. Data were analysed using multiple comparison ANOVA with Tukey-Kramer

571 post hoc tests or unpaired t-test with Mann-Whitney test, as noted in the Results section.

572 The null hypothesis was rejected at a 0.05 level of significance. 


\section{List of Abbreviations:}

575 MeDUsA : a method for quantification of degeneration using fly axons

576 ND: Neurodegenerative disease

577 REP: rough eye phenotype

578 SCA3: Spinocerebellar ataxia type 3

579 PD: Parkinson's disease

580 ALS: amyotrophic lateral sclerosis

581 CNN: convolutional neural network

582 RNAi: RNA interference

583 SCA31: spinocerebellar ataxia type 31

584 FTLD: frontotemporal lobar degeneration

585 WD: Wallerian degeneration

586

\section{Declarations}

588 Ethics approval and consent to participate

$589 \quad$ Not applicable

590

\section{Consent for publication}

592 Not applicable

$594 \quad$ Availability of data and materials

595 The software documentation for MeDUsA can be found at

596 https://github.com/SugieLab/MeDUsA. 
597 The datasets used and/or analyzed during the current study are available from the

598 corresponding authors on reasonable request.

599

600 Competing interests

601 The authors declare that we have no competing interests.

602

603 Funding

604 This work was supported in part by grants from the Ministry of Education, Culture,

605 Sports, Science and Technology of Japan (\#18K14835, \#18J00367 and \#21K15619 to

606 YNI, \#21K06184 to SHS, \#17H05699 to YNA, \#16H06457 and \#21H02483 to TS,

607 \#17H04983, \#19K22592 and \#21H02837 to AS), grants for Strategic Research Program

608 for Brain Sciences from Japan Agency for Medical Research and Development, Japan

609 (\#JP20dm0107061 to YNA), Takeda Science Foundation Takeda Visionary Research

610 Grant to T.S., and Takeda science foundation life science research grant to AS. DZNE

611 core funding to G.T.

612

\section{Authors' contributions}

614 YNI and AS designed and organized the study.

615 YNI and AS performed immunohistochemistry, and eye imaging.

616 YNI, AS and KD performed data analysis.

617 HK established the software.

$618 \mathrm{JO}, \mathrm{SHS}$, and TS designed the screen experiment and JO performed the experiment and

619 data analysis.

620 YNA generated transgenic flies. 
622 All authors read and approved the final manuscript.

623

\section{$624 \underline{\text { Acknowledgements }}$}

625 We would like to acknowledge that Dr. Zipursky, Dr. Feany, Dr. Lee and Dr. Littleton 626 have provided us with transgenic fly strains. We would like to thank Ms. Nozaki for 627 helping us to quantify REP.

628

\section{Figure Legends:}

630 Figure 1. Rough eye and axonal degeneration phenotypes for evaluating neurodegeneration.

632

(A) Dorsal schematic of the visual system in Drosophila. The axons of photoreceptors $\mathrm{R} 7$ and $\mathrm{R} 8$ project from the retina through the lamina to the medulla. (B) The process of 634 exploring factors that mitigate TDP-43 toxicity using a combination of rough eye and 635 axonal degeneration phenotypic observations. (C) Candidate genes that suppress the rough eye phenotype (REP) and their involvement in axonal toxicity. In knockdown

637 screening, six genes suppressed REP and eight genes mildly suppressed it. However, the 638 degree of REP and severity of R axon degeneration were not consistent. Yellow arrows 639 indicate fragmented axons. Scale bar $=20 \mu m$. (D) Quantification of the ratio of axonal 640 degeneration. ${ }^{* *} p<0.001$. Chi-square test was performed between the control and each 641 knockdown. 
644 (A) Schematic of the visual system in Drosophila. (B) All R7 and R8 axon terminals

645 projecting to the medulla. $\mathrm{R}$ axons were stained with anti-Chaoptin, which is a

646 photoreceptor-specific antibody (green). Scale bar $=20 \mu m$. (C) Process flow of

647 generating a surface mask. First, for each slice of the $\mathrm{z}$-stack image, three slices $(\mathrm{z}-1$,

$648 \mathrm{z}$ and $\mathrm{z}+1$ ) are merged by adding the previous and next slice to create an RGB image.

649 The RGB image is inputted into the training model to generate the RGB mask. Finally,

650 only the channel corresponding to the central slice is extracted to obtain the final mask.

651 (D) Process for axon terminal detection. Using the obtained surface mask stack and

652 original image stack, the surface mask region is extracted from the original image for

653 each slice, and only the signal at the axon end is extracted. Axon terminals are then

654 detected and quantified from the obtained 3D volume.

655

656

Figure 3. Toxicity evaluation of representative ND causative factors using MeDUsA.

657

(A-J) The R axon terminals stained with anti-Chaoptin and extracted using MeDUsA.

658

(A) Control and photoreceptor expression of

(B) $\alpha \operatorname{Syn}^{\mathrm{WT}}$,

(C) $\alpha \operatorname{Syn}^{\mathrm{A} 53 \mathrm{~T}}$,

(D) $\mathrm{Tau}^{\mathrm{WT}}$,

$\mathrm{Tau}^{\mathrm{R} 406 \mathrm{~W}}$,

(F) $\mathrm{Tau}^{\mathrm{S} 2 \mathrm{~A}}$

(G) TDP-43 ${ }^{\mathrm{WT}}$,

(H) TDP-43 ${ }^{\mathrm{A} 315 \mathrm{~T}}$,

, (I) $\mathrm{HTT}^{\mathrm{Q} 0}$ and (J) $\mathrm{HTT}^{\mathrm{Q} 128}$. Scale

660

bar $=20 \mu \mathrm{m} .(\mathrm{K})$ The number of axons expressing each pathogenic factor was predicted.

661

$* * * * p<0.0001, * * * p<0.001$ and ns $(p>0.05)$. Data were analysed using multiple

662

comparison ANOVA with Tukey-Kramer post hoc tests. Error bars show the standard

663

error of the mean.

664

Figure 4. Comparison between manual quantification and the MeDUsA system.

666 (A) The axonal numbers quantified by manual measurements using the same dataset shown in Figure 3 K. $* * * * p<0.00001, * * * p<0.001, * * p<0.01,{ }^{*} p<0.05$ and ns $(p>$ 
670 number predicted by MeDUsA to those measured manually for each genotype. (C) The

671 correlation between the individual values measured manually and the individual values

672 predicted by MeDUsA. (D) The correlation between the average of each genotype measured manually versus those predicted by MeDUsA. $\mathrm{R}^{2}$, coefficient of determination.

674

675 Figure 5. Ectopic expression of causative genes of NDs in fly eye causing REP, but

676 inconsistent with axonal degeneration.

677 (A-J) Representative bright-field microscope images of (A) control or fly eyes overexpressing (B) $\mathrm{SNCA}^{\mathrm{WT}}$, (C) $\mathrm{SNCA}^{\mathrm{A} 53 \mathrm{~T}}$, (D) $\mathrm{Tau}^{\mathrm{WT}}$, (E) $\mathrm{Tau}^{\mathrm{R} 406 \mathrm{~W}},(\mathrm{~F}) \mathrm{Tau}^{\mathrm{S} 2 \mathrm{~A}},(\mathrm{G})$

679 TDP-43 ${ }^{\mathrm{WT}}$, (H) TDP-43 ${ }^{\mathrm{A} 315 \mathrm{~T}}$, (I) HTT ${ }^{\mathrm{Q} 0}$ and (J) HTT ${ }^{\mathrm{Q} 128}$ using the GMR-Gal4 driver. (K)

680 Graph representing the phenotypic score (P score) of each genotype calculated using

681 Flynotyper. ${ }^{* * *} p<0.0001, * * p<0.01$ and ns $(p>0.05)$. Data were analysed using 682 multiple comparison ANOVA with Tukey-Kramer post hoc tests. Error bars show the 683 standard error of the mean.

684

\section{$685 \underline{\text { Reference }}$}

686

687 1. Dugger BN, Dickson DW. Pathology of Neurodegenerative Diseases. Csh Perspect Biol. 2017;9:a028035. 
690 Expanded Polyglutamine Protein Forms Nuclear Inclusions and Causes Neural

691 Degeneration in Drosophila. Cell. 1998;93:939-49.

692 3. Jackson GR, Salecker I, Dong X, Yao X, Arnheim N, Faber PW, et al.

693 Polyglutamine-Expanded Human Huntingtin Transgenes Induce Degeneration of

694 Drosophila Photoreceptor Neurons. Neuron. 1998;21:633-42.

695 4. Feany MB, Bender WW. A Drosophila model of Parkinson's disease. Nature.

$6962000 ; 404: 394-8$.

697 5. Wittmann CW, Wszolek MF, Shulman JM, Salvaterra PM, Lewis J, Hutton M, et al.

698 Tauopathy in Drosophila: neurodegeneration without neurofibrillary tangles. Science.

$699 \quad 2001 ; 293: 711-4$.

700 6. Chouhan AK, Guo C, Hsieh Y-C, Ye H, Senturk M, Zuo Z, et al. Uncoupling

701 neuronal death and dysfunction in Drosophila models of neurodegenerative disease.

702 Acta Neuropathologica Commun. 2016;4:62.

703 7. Elden AC, Kim H-J, Hart MP, Chen-Plotkin AS, Johnson BS, Fang X, et al. Ataxin-2

704 intermediate-length polyglutamine expansions are associated with increased risk for

705 ALS. Nature. 2010;466:1069-75.

706 8. Brand AH, Perrimon N. Targeted gene expression as a means of altering cell fates

707 and generating dominant phenotypes. Development. 1993;118:401-15. 
708 9. Blard O, Feuillette S, Bou J, Chaumette B, Frébourg T, Campion D, et al.

709 Cytoskeleton proteins are modulators of mutant tau-induced neurodegeneration in

710 Drosophila. Hum Mol Genet. 2007;16:555-66.

711 10. Chen X, Li Y, Huang J, Cao D, Yang G, Liu W, et al. Study of tauopathies by

712 comparing Drosophila and human tau in Drosophila. Cell Tissue Res. 2007;329:169-

71378.

714 11. Ambegaokar SS, Jackson GR. Functional genomic screen and network analysis

715 reveal novel modifiers of tauopathy dissociated from tau phosphorylation. Hum Mol

716 Genet. 2011;20:4947-77.

717 12. M'Angale PG, Staveley BE. The Bcl-2 homologue Buffy rescues $\alpha$-synuclein-

718 induced Parkinson disease-like phenotypes in Drosophila. Bmc Neurosci. 2016;17:24.

719 13. Alexopoulou Z, Lang J, Perrett RM, Elschami M, Hurry MED, Kim HT, et al.

720 Deubiquitinase Usp8 regulates $\alpha$-synuclein clearance and modifies its toxicity in Lewy

721 body disease. Proc National Acad Sci. 2016;113:E4688-97.

722 14. Davies SE, Hallett PJ, Moens T, Smith G, Mangano E, Kim HT, et al. Enhanced

723 ubiquitin-dependent degradation by Nedd4 protects against $\alpha$-synuclein accumulation

724 and toxicity in animal models of Parkinson's disease. Neurobiol Dis. 2014;64:79-87.

725 15. Miura E, Hasegawa T, Konno M, Suzuki M, Sugeno N, Fujikake N, et al. VPS35

726 dysfunction impairs lysosomal degradation of $\alpha$-synuclein and exacerbates

727 neurotoxicity in a Drosophila model of Parkinson's disease. Neurobiol Dis. 2014;71:172813. 
17. Kim H-J, Raphael AR, LaDow ES, McGurk L, Weber RA, Trojanowski JQ, et al.

732 Therapeutic modulation of eIF $2 \alpha$ phosphorylation rescues TDP-43 toxicity in amyotrophic lateral sclerosis disease models. Nat Genet. 2014;46:152-60. Drosophila junctophilin gene is functionally equivalent to its four mammalian counterparts and is a modifier of a Huntingtin poly-Q expansion and the Notch pathway. Dis Model Mech. 2017;11:dmm029082. interacting proteins are genetic modifiers of neurodegeneration. PLoS Genetics. 2007;3:e82.

744 21. Shim K-H, Kim S-H, Hur J, Kim D-H, Demirev AV, Yoon S-Y. Small-molecule

745 drug screening identifies drug Ro 31-8220 that reduces toxic phosphorylated tau in

746 Drosophila melanogaster. Neurobiol Dis. 2019;130:104519.

747 22. Lin Y, Xue J, Deng J, He H, Luo S, Chen J, et al. Neddylation activity modulates

748 the neurodegeneration associated with fragile $\mathrm{X}$ associated tremor/ataxia syndrome

749 (FXTAS) through regulating Sima. Neurobiol Dis. 2020;143:105013. 
751 image analysis method to measure regularity in biological patterns: a case study in a

752 Drosophila neurodegenerative model. Molecular neurodegeneration. 2015;10:9.

753 24. Iyer J, Wang Q, Le T, Pizzo L, Grönke S, Ambegaokar SS, et al. Quantitative

754 Assessment of Eye Phenotypes for Functional Genetic Studies Using Drosophila

755 melanogaster. G3 (Bethesda, Md). 2016;6:1427-37.

757 Neurodegenerative Disease. Genetics. 2015;201:377-402.

758

759

760

761

762

763

764

765

766

767

30. Çiçek Ö, Abdulkadir A, Lienkamp SS, Brox T, Ronneberger O. 3D U-Net: Learning

26. Bhattacharya MRC, Gerdts J, Naylor SA, Royse EX, Ebstein SY, Sasaki Y, et al. A model of toxic neuropathy in Drosophila reveals a role for MORN4 in promoting axonal degeneration. Journal of Neuroscience. 2012;32:5054-61.

27. Sreedharan J, Neukomm LJ, Brown RH, Freeman MR. Age-Dependent TDP-43-

Mediated Motor Neuron Degeneration Requires GSK3, hat-trick, and xmas-2. Curr Biol. 2015;25:2130-6.

28. Moen E, Bannon D, Kudo T, Graf W, Covert M, Valen DV. Deep learning for cellular image analysis. Nat Methods. 2019;16:1233-46.

29. Ronneberger O, Fischer P, Brox T. U-Net: Convolutional Networks for Biomedical Image Segmentation. Lect Notes Comput Sc. 2015;234-41.

Dense Volumetric Segmentation from Sparse Annotation. Medical Image Computing 
and Computer-Assisted Intervention - MICCAI 2016, 19th International Conference,

771 Athens, Greece, October 17-21, 2016, Proceedings, Part II. 2016. p. 424-32.

772 31. Falk T, Mai D, Bensch R, Çiçek Ö, Abdulkadir A, Marrakchi Y, et al. U-Net: deep

773 learning for cell counting, detection, and morphometry. Nat Methods. 2019;16:67-70.

774 32. Fischer CA, Besora-Casals L, Rolland SG, Haeussler S, Singh K, Duchen M, et al.

775 MitoSegNet: Easy-to-use Deep Learning Segmentation for Analyzing Mitochondrial

776 Morphology. iScience. 2020;23:101601.

777 33. Long F. Microscopy cell nuclei segmentation with enhanced U-Net. Bmc

778 Bioinformatics. 2020;21:8.

779 34. Richard M, Doubková K, Nitta Y, Kawai H, Sugie A, Tavosanis G. A quantitative

780 model of sporadic axonal degeneration in the Drosophila visual system. bioRxiv. 2021;

781 35. Sang T-K, Jackson GR. Drosophila models of neurodegenerative disease. Neurorx.

$782 \quad 2005 ; 2: 438-46$.

783 36. Deerlin VMV, Leverenz JB, Bekris LM, Bird TD, Yuan W, Elman LB, et al.

784 TARDBP mutations in amyotrophic lateral sclerosis with TDP-43 neuropathology: a

785 genetic and histopathological analysis. Lancet Neurology. 2008;7:409-16.

786 37. Newsome TP, Asling B, Dickson BJ. Analysis of Drosophila photoreceptor axon

787 guidance in eye-specific mosaics. Development. 2000;127:851-60. 
795 Synaptic Modulation of Drosophila melanogaster Photoreceptors after Exposure to

796 Prolonged Light. J Vis Exp. 2017;

797 41. Spillantini MG, Schmidt ML, Lee VM-Y, Trojanowski JQ, Jakes R, Goedert M. $\alpha$ 798 Synuclein in Lewy bodies. Nature. 1997;388:839-40.

799 42. Polymeropoulos MH, Lavedan C, Leroy E, Ide SE, Dehejia A, Dutra A, et al.

800 Mutation in the $\alpha$-Synuclein Gene Identified in Families with Parkinson9s Disease.

801 Science. 1997;276:2045-7.

803 of missense and 5'-splice-site mutations in tau with the inherited dementia FTDP-17.

804 Nature. 1998;393:702-5.

805 44. Gitcho MA, Baloh RH, Chakraverty S, Mayo K, Norton JB, Levitch D, et al. TDP-

80643 A315T mutation in familial motor neuron disease. Ann Neurol. 2008;63:535-8. 
807 45. Chen X, Peterson J, Nachman RJ, Ganetzky B. Drosulfakinin activates CCKLR-

808 17D1 and promotes larval locomotion and escape response in Drosophila. Fly.

$809 \quad 2012 ; 6: 290-7$.

810 46. Williams MJ, Goergen P, Rajendran J, Zheleznyakova G, Hägglund MG, Perland E,

811 et al. Obesity-Linked Homologues TfAP-2 and Twz Establish Meal Frequency in

812 Drosophila melanogaster. Plos Genet. 2014;10:e1004499.

813 47. Agrawal P, Kao D, Chung P, Looger LL. The neuropeptide Drosulfakinin regulates

814 social isolation-induced aggression in Drosophila. J Exp Biol. 2020;223:jeb207407.

815 48. Wu F, Deng B, Xiao N, Wang T, Li Y, Wang R, et al. A neuropeptide regulates

816 fighting behavior in Drosophila melanogaster. Elife. 2020;9:e54229.

817 49. Chen X, Ganetzky B. A neuropeptide signaling pathway regulates synaptic growth 818 in Drosophila. J Cell Biol. 2012;196:529-43.

819 50. Fogarty MJ, Klenowski PM, Lee JD, Drieberg-Thompson JR, Bartlett SE, Ngo ST,

820 et al. Cortical synaptic and dendritic spine abnormalities in a presymptomatic TDP-43

821 model of amyotrophic lateral sclerosis. Sci Rep-uk. 2016;6:37968.

822 51. Jiang T, Handley E, Brizuela M, Dawkins E, Lewis KEA, Clark RM, et al.

823 Amyotrophic lateral sclerosis mutant TDP-43 may cause synaptic dysfunction through

824 altered dendritic spine function. Dis Model Mech. 2019;12:dmm038109.

825 52. Breen TR, Lucchesi JC. Analysis of the dosage compensation of a specific transcript

826 in Drosophila melanogaster. Genetics. 1986;112:483-91. 
828 Interacting Proteins Reveals Strong Association with RNA Splicing and Translation

829 Machinery. J Proteome Res. 2010;9:1104-20.

830 54. Kim SH, Shanware NP, Bowler MJ, Tibbetts RS. Amyotrophic Lateral Sclerosis-

831 associated Proteins TDP-43 and FUS/TLS Function in a Common Biochemical

832 Complex to Co-regulate HDAC6 mRNA*. J Biol Chem. 2010;285:34097-105.

833 55. Ling S-C, Albuquerque CP, Han JS, Lagier-Tourenne C, Tokunaga S, Zhou H, et al.

834 ALS-associated mutations in TDP-43 increase its stability and promote TDP-43

835 complexes with FUS/TLS. Proc National Acad Sci. 2010;107:13318-23.

836 56. Wang J-W, Brent JR, Tomlinson A, Shneider NA, McCabe BD. The ALS-

837 associated proteins FUS and TDP-43 function together to affect Drosophila locomotion

838 and life span. J Clin Invest. 2011;121:4118-26.

839 57. Kabashi E, Bercier V, Lissouba A, Liao M, Brustein E, Rouleau GA, et al. FUS and

840 TARDBP but Not SOD1 Interact in Genetic Models of Amyotrophic Lateral Sclerosis.

$841 \quad$ Plos Genet. 2011;7:e1002214.

842 58. Lee S, Wang J-W, Yu W, Lu B. Phospho-dependent ubiquitination and degradation

843 of PAR-1 regulates synaptic morphology and tau-mediated A $\beta$ toxicity in Drosophila.

844 Nature Communications. 2012;3:1312-12.

845 59. Burré J, Sharma M, Südhof TC. Cell Biology and Pathophysiology of $\alpha$-Synuclein.

846 Csh Perspect Med. 2018;8:a024091. 
847 60. Orimo S, Uchihara T, Nakamura A, Mori F, Kakita A, Wakabayashi K, et al.

848 Axonal $\alpha$-synuclein aggregates herald centripetal degeneration of cardiac sympathetic

849 nerve in Parkinson's disease. Brain. 2008;131:642-50.

850 61. Volpicelli-Daley LA, Luk KC, Patel TP, Tanik SA, Riddle DM, Stieber A, et al.

851 Exogenous $\alpha$-Synuclein Fibrils Induce Lewy Body Pathology Leading to Synaptic

852 Dysfunction and Neuron Death. Neuron. 2011;72:57-71.

853 62. Lashuel HA, Overk CR, Oueslati A, Masliah E. The many faces of $\alpha$-synuclein:

854 from structure and toxicity to therapeutic target. Nature Publishing Group. 2013;14:38-

85548.

856 63. Kouroupi G, Taoufik E, Vlachos IS, Tsioras K, Antoniou N, Papastefanaki F, et al.

857 Defective synaptic connectivity and axonal neuropathology in a human iPSC-based

858 model of familial Parkinson's disease. Proc National Acad Sci. 2017;114:E3679-88.

859 64. Bengoa-Vergniory N, Roberts RF, Wade-Martins R, Alegre-Abarrategui J. Alpha-

860 synuclein oligomers: a new hope. Acta Neuropathologica. 2017;134:819-38.

861 65. Kolarova M, García-Sierra F, Bartos A, Ricny J, Ripova D. Structure and Pathology

862 of Tau Protein in Alzheimer Disease. Int J Alzheimer's Dis. 2012;2012:731526.

863 66. Dayanandan R, Slegtenhorst MV, Mack TGA, Ko L, Yen S-H, Leroy K, et al.

864 Mutations in tau reduce its microtubule binding properties in intact cells and affect its

865 phosphorylation. Febs Lett. 1999;446:228-32. 
867 Nagashima K, et al. Molecular Analysis of Mutant and Wild-Type Tau Deposited in the

868 Brain Affected by the FTDP-17 R406W Mutation. Am J Pathology. 2001;158:373-9.

869 68. Ikeda M, Kawarai T, Kawarabayashi T, Matsubara E, Murakami T, Sasaki A, et al.

870 Accumulation of Filamentous Tau in the Cerebral Cortex of Human Tau R406W

871 Transgenic Mice. Am J Pathology. 2005;166:521-31.

872 69. Nakamura M, Shiozawa S, Tsuboi D, Amano M, Watanabe H, Maeda S, et al.

873 Pathological Progression Induced by the Frontotemporal Dementia-Associated R406W

874 Tau Mutation in Patient-Derived iPSCs. Stem Cell Rep. 2019;13:684-99.

875 70. Jackson GR, Wiedau-Pazos M, Sang T-K, Wagle N, Brown CA, Massachi S, et al.

876 Human Wild-Type Tau Interacts with wingless Pathway Components and Produces

877 Neurofibrillary Pathology in Drosophila. Neuron. 2002;34:509-19.

878 71. Povellato G, Tuxworth RI, Hanger DP, Tear G. Modification of the Drosophila 879 model of in vivo Tau toxicity reveals protective phosphorylation by GSK3 $\beta$. Biol Open. $880 \quad 2013 ; 3: 1-11$.

881 72. Neumann M, Sampathu DM, Kwong LK, Truax AC, Micsenyi MC, Chou TT, et al.

882 Ubiquitinated TDP-43 in Frontotemporal Lobar Degeneration and Amyotrophic Lateral 883 Sclerosis. Science. 2006;314:130-3.

884 73. Prasad A, Bharathi V, Sivalingam V, Girdhar A, Patel BK. Molecular Mechanisms 885 of TDP-43 Misfolding and Pathology in Amyotrophic Lateral Sclerosis. Front Mol

$886 \quad$ Neurosci. 2019;12:25. 
888 loss of function of ALS-related mutations of TARDBP (TDP-43) cause motor deficits

889 in vivo. Hum Mol Genet. 2010;19:671-83.

890 75. Xu Y-F, Gendron TF, Zhang Y-J, Lin W-L, D’Alton S, Sheng H, et al. Wild-Type

891 Human TDP-43 Expression Causes TDP-43 Phosphorylation, Mitochondrial

892 Aggregation, Motor Deficits, and Early Mortality in Transgenic Mice. J Neurosci.

$893 \quad 2010 ; 30: 10851-9$.

894 76. Ash PEA, Zhang Y-J, Roberts CM, Saldi T, Hutter H, Buratti E, et al. Neurotoxic

895 effects of TDP-43 overexpression in C. elegans. Hum Mol Genet. 2010;19:3206-18.

896 77. Diaper DC, Adachi Y, Sutcliffe B, Humphrey DM, Elliott CJH, Stepto A, et al. Loss

897 and gain of Drosophila TDP-43 impair synaptic efficacy and motor control leading to

898 age-related neurodegeneration by loss-of-function phenotypes. Hum Mol Genet.

$899 \quad 2013 ; 22: 1539-57$.

900 78. Li Y, Ray P, Rao EJ, Shi C, Guo W, Chen X, et al. A Drosophila model for TDP-43

901 proteinopathy. Proc National Acad Sci. 2010;107:3169-74.

902 79. Guo W, Chen Y, Zhou X, Kar A, Ray P, Chen X, et al. An ALS-associated mutation

903 affecting TDP-43 enhances protein aggregation, fibril formation and neurotoxicity. Nat

904 Struct Mol Biol. 2011;18:822-30.

907 ALS. Hum Mol Genet. 2011;20:2308-21. 
908 81. Roos RA. Huntington's disease: a clinical review. Orphanet J Rare Dis. 2010;5:40.

909 82. Gatto RG, Chu Y, Ye AQ, Price SD, Tavassoli E, Buenaventura A, et al. Analysis

910 of $\mathrm{YFP}(\mathrm{J} 16)-\mathrm{R} 6 / 2$ reporter mice and postmortem brains reveals early pathology and

911 increased vulnerability of callosal axons in Huntington's disease. Hum Mol Genet.

$912 \quad 2015 ; 24: 5285-98$.

913 83. Zhang S, Binari R, Zhou R, Perrimon N. A Genomewide RNA Interference Screen

914 for Modifiers of Aggregates Formation by Mutant Huntingtin in Drosophila. Genetics.

$915 \quad 2010 ; 184: 1165-79$.

916 84. Ferri A, Sanes JR, Coleman MP, Cunningham JM, Kato AC. Inhibiting Axon

917 Degeneration and Synapse Loss Attenuates Apoptosis and Disease Progression in a

918 Mouse Model of Motoneuron Disease. Curr Biol. 2003;13:669-73.

919 85. Sajadi A, Schneider BL, Aebischer P. Wlds-Mediated Protection of Dopaminergic

920 Fibers in an Animal Model of Parkinson Disease. Curr Biol. 2004;14:326-30.

921 86. Velde CV, Garcia ML, Yin X, Trapp BD, Cleveland DW. The neuroprotective

922 factor Wlds does not attenuate mutant SOD1-mediated motor neuron disease. Neuromol

923 Med. 2004;5:193-203.

924 87. Haberl MG, Churas C, Tindall L, Boassa D, Phan S, Bushong EA, et al.

925 CDeep3M-Plug-and-Play cloud-based deep learning for image segmentation. Nat

926 Methods. 2018;15:677-80. 
928 Representation of Loss of Eye Regularity in a Drosophila Neurodegenerative Model.

929 Front Neurosci-switz. 2020;14:516.

930 89. Kayasandik CB, Ru W, Labate D. A multistep deep learning framework for the

931 automated detection and segmentation of astrocytes in fluorescent images of brain

932 tissue. Sci Rep-uk. 2020;10:5137.

934 Machine learning-based classification of mitochondrial morphology in primary neurons

935 and brain. Sci Rep-uk. 2021;11:5133.

936 91. Berger-Müller S, Sugie A, Takahashi F, Tavosanis G, Hakeda-Suzuki S, Suzuki T.

937 Assessing the Role of Cell-Surface Molecules in Central Synaptogenesis in the

938 Drosophila Visual System. Plos One. 2013;8:e83732.

939 92. Gao S, Takemura S, Ting C-Y, Huang S, Lu Z, Luan H, et al. The Neural Substrate

940 of Spectral Preference in Drosophila. Neuron. 2008;60:328-42.

941 93. Chen Y, Akin O, Nern A, Tsui CYK, Pecot MY, Zipursky SL. Cell-type-Specific

942 Labeling of Synapses In Vivo through Synaptic Tagging with Recombination. Neuron.

$943 \quad 2014 ; 81: 280-93$.

944 94. Lee W-CM, Yoshihara M, Littleton JT. Cytoplasmic aggregates trap polyglutamine-

945 containing proteins and block axonal transport in a Drosophila model of Huntington's

946 disease. P Natl Acad Sci Usa. 2004;101:3224-9. 
947 95. Saitoh Y, Fujikake N, Okamoto Y, Popiel HA, Hatanaka Y, Ueyama M, et al. p62

948 Plays a Protective Role in the Autophagic Degradation of Polyglutamine Protein

949 Oligomers in Polyglutamine Disease Model Flies*. J Biol Chem. 2015;290:1442-53.

950 96. Schindelin J, Arganda-Carreras I, Frise E, Kaynig V, Longair M, Pietzsch T, et al.

951 Fiji: an open-source platform for biological-image analysis. Nature Methods.

$952 \quad 2012 ; 9: 676-82$. 


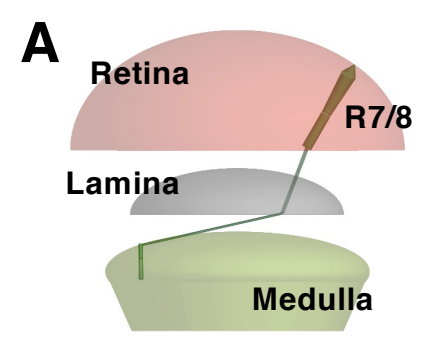

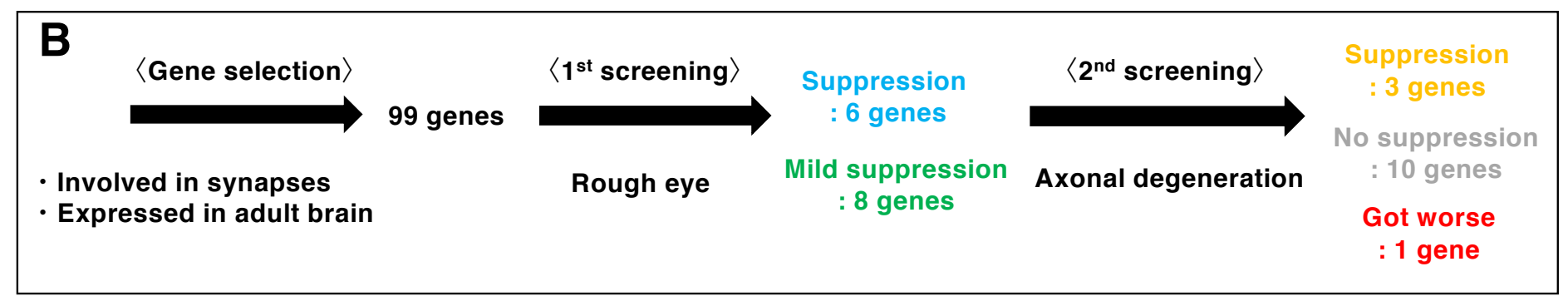
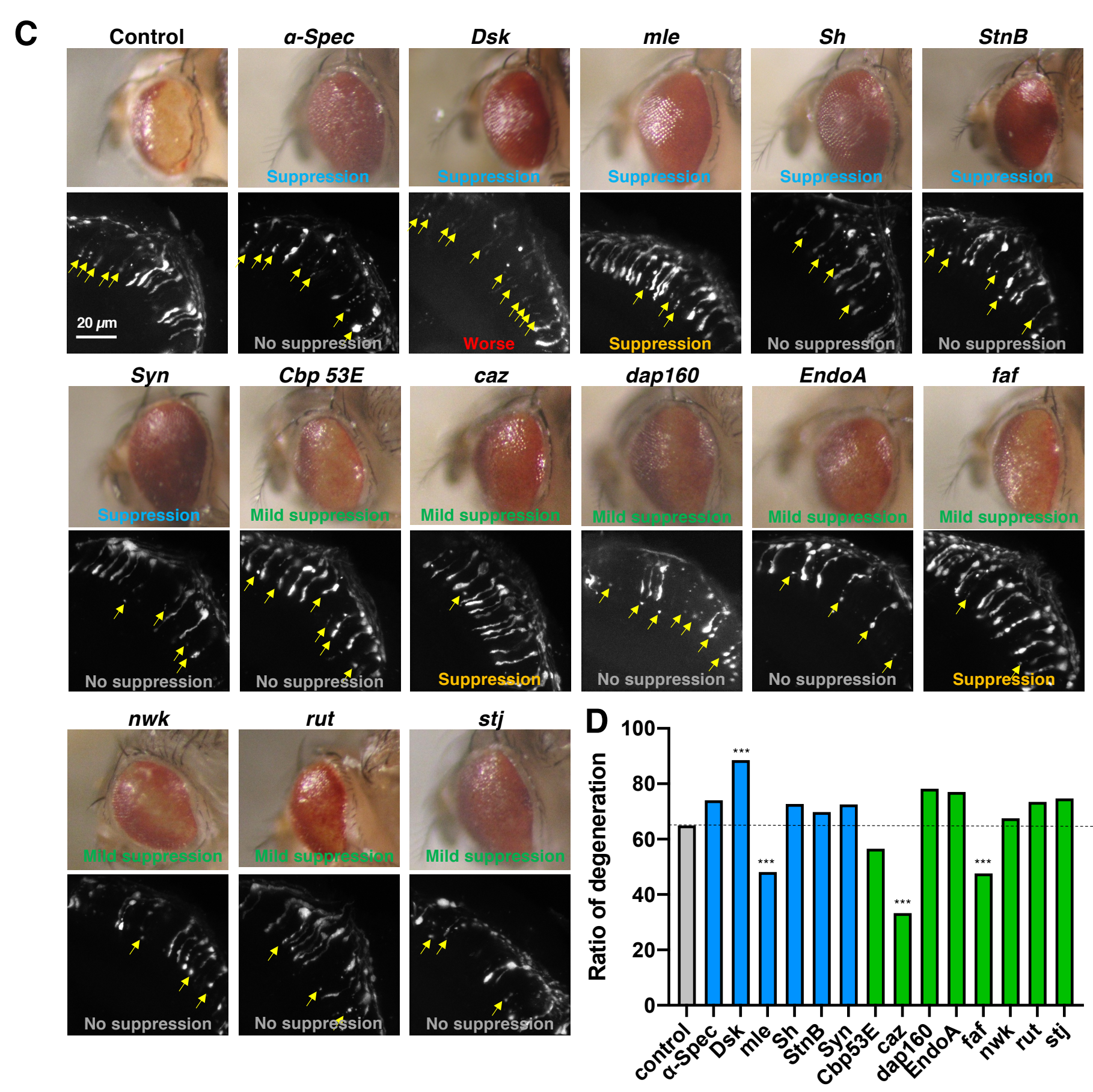

Fig. 1 


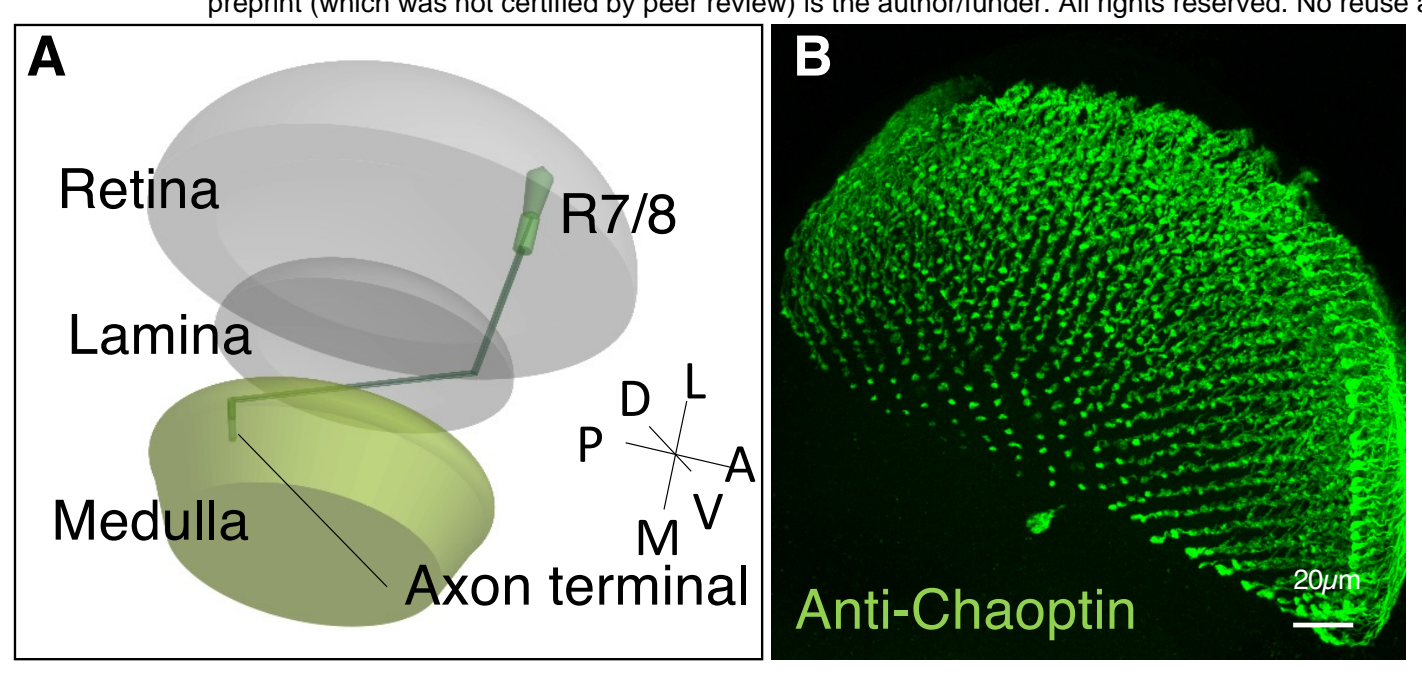

\section{C}

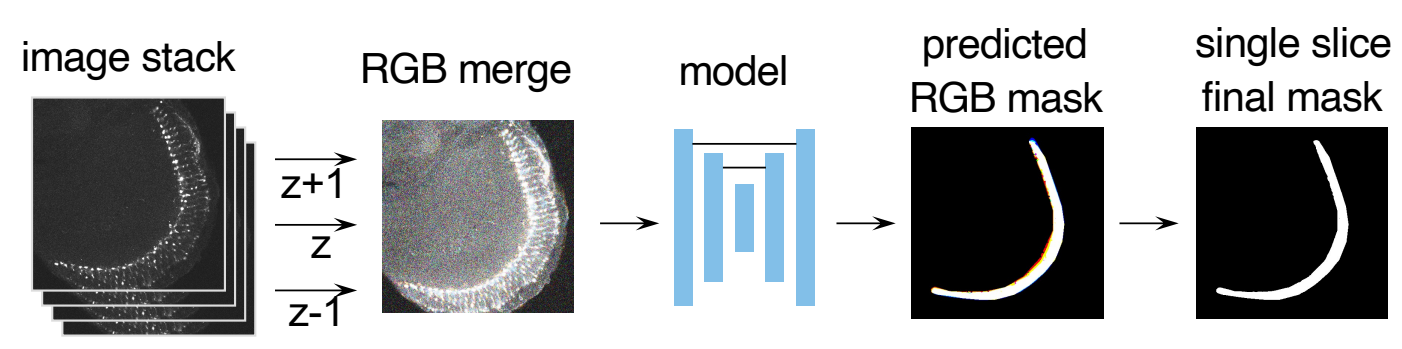

D

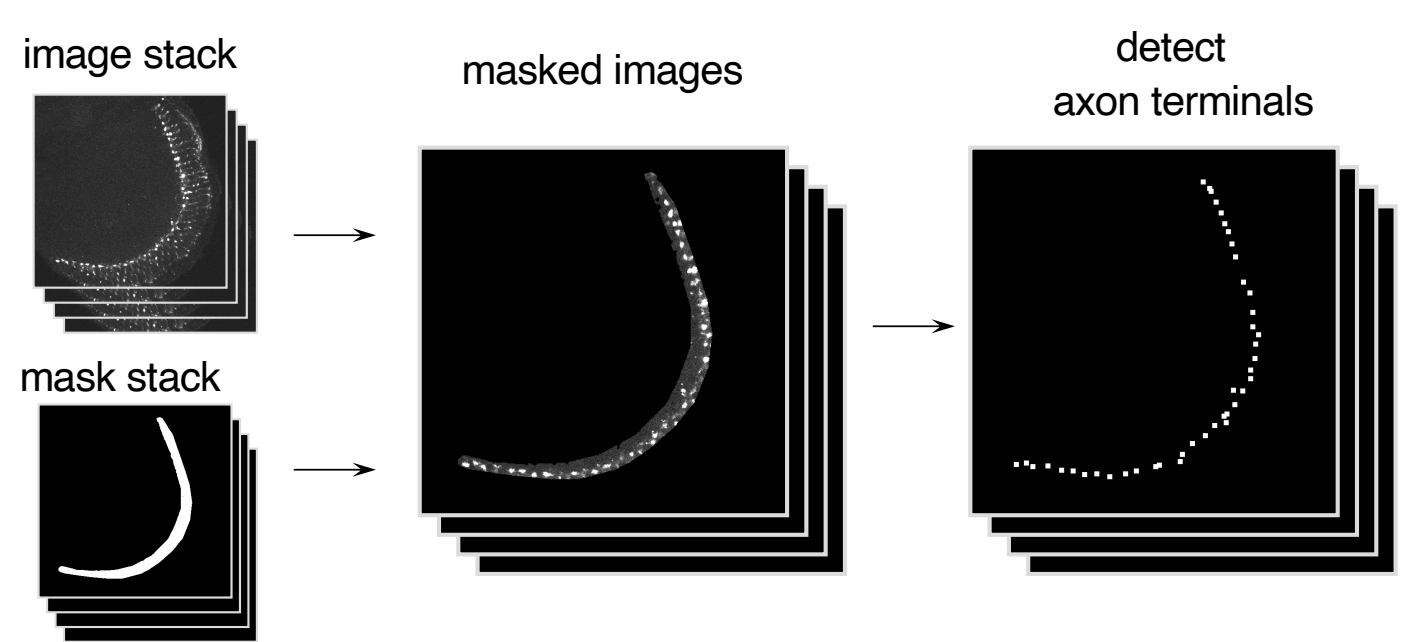

Fig. 2 

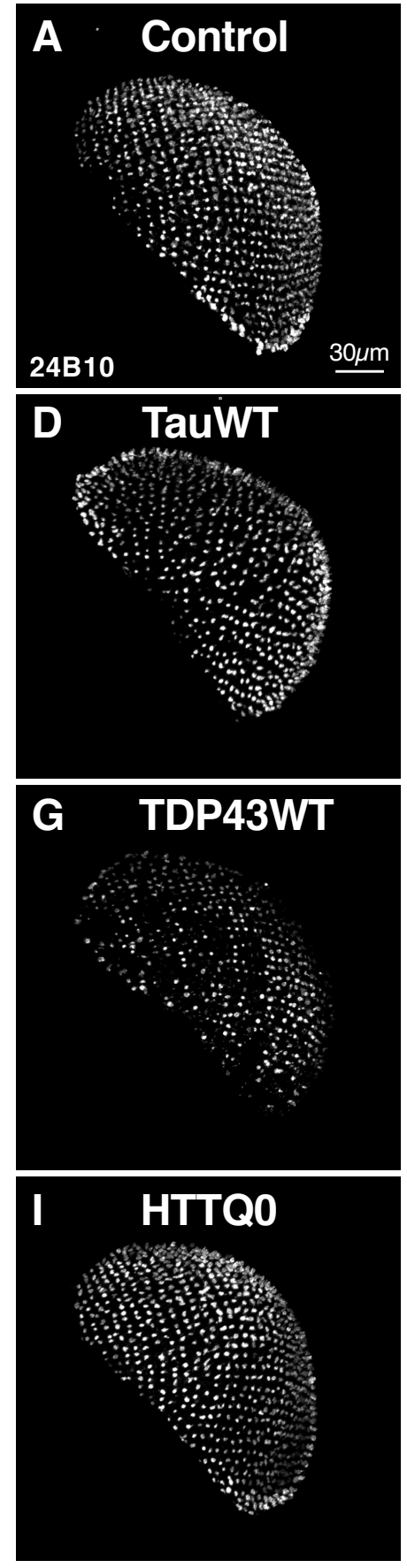
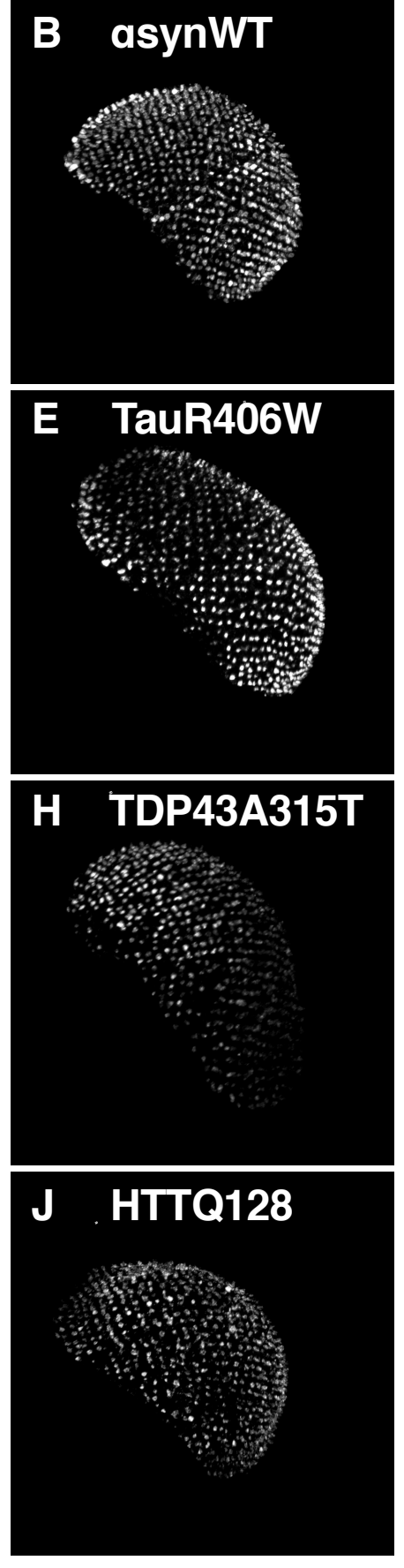
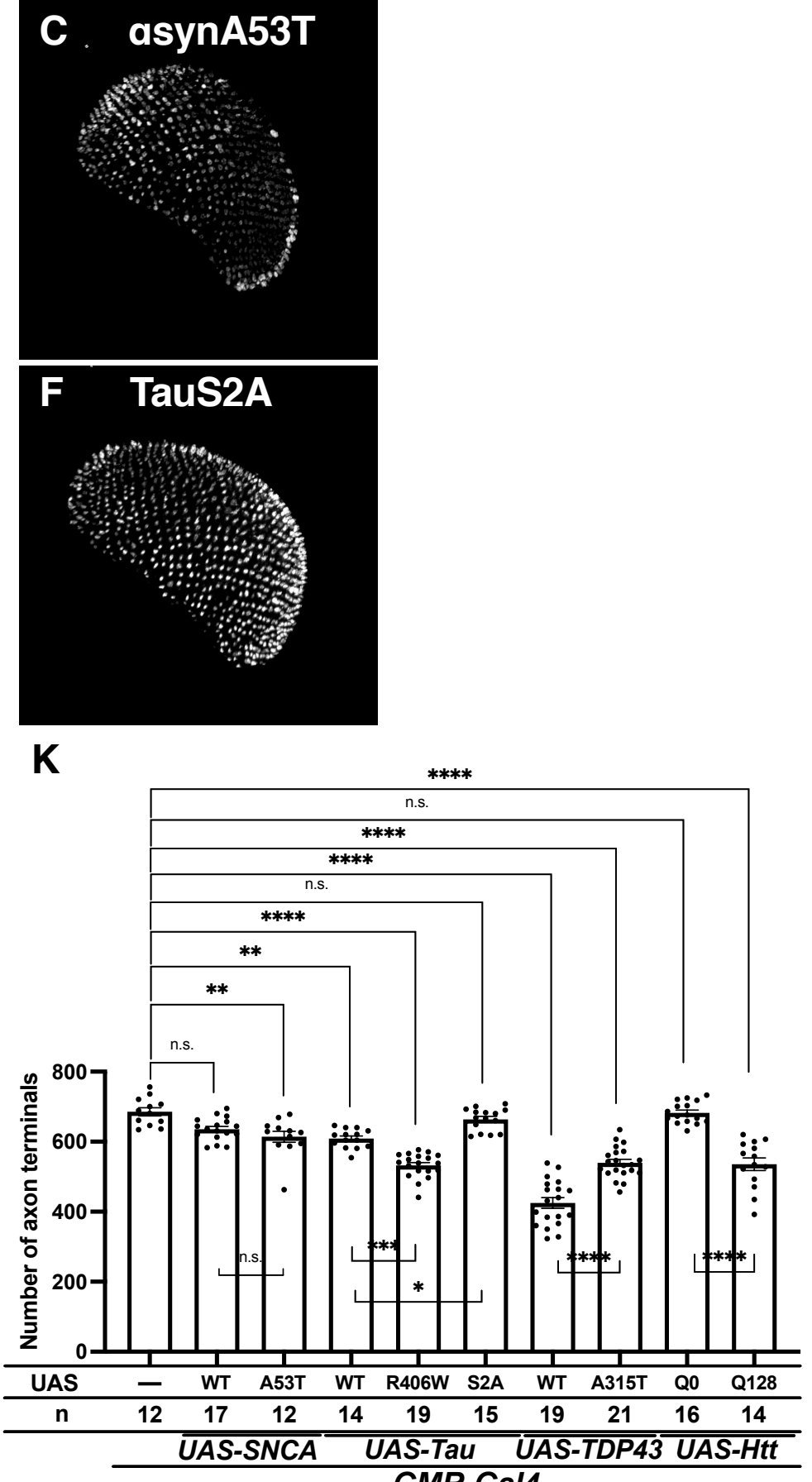
bioRxiv preprint doi: https://doi.org/10.1101/2021.10.25.465674; this version posted October 26, 2021. The copyright holder for this

preprint (which was not certified by peer review) is the author/funder. All rights reserved. No reuse allowed without permission.

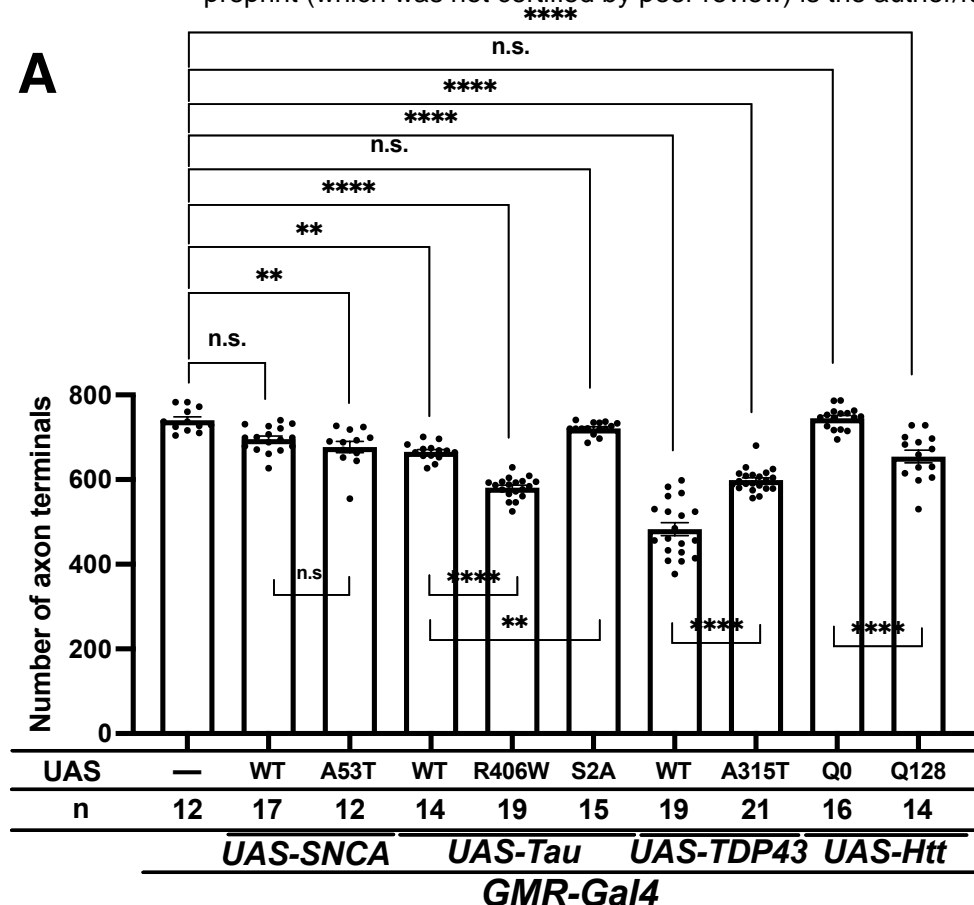

B

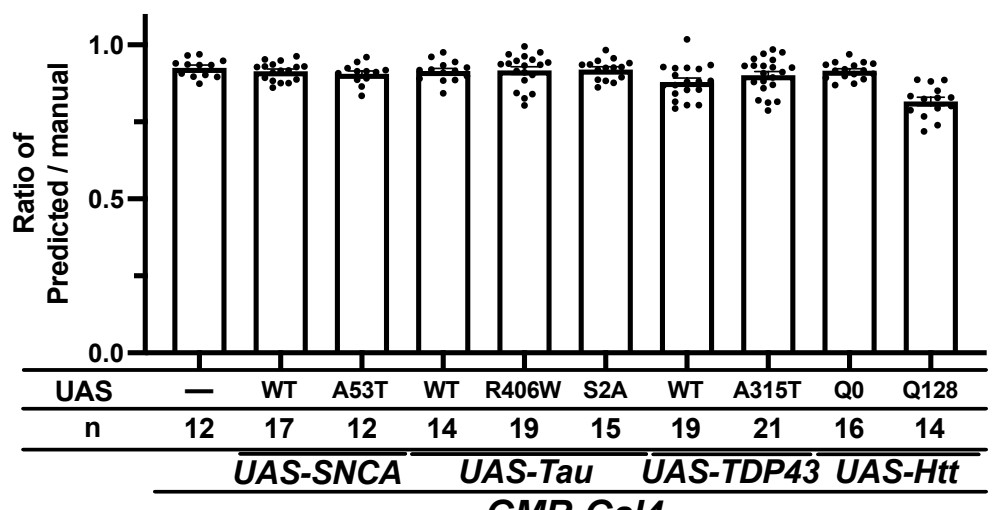

C

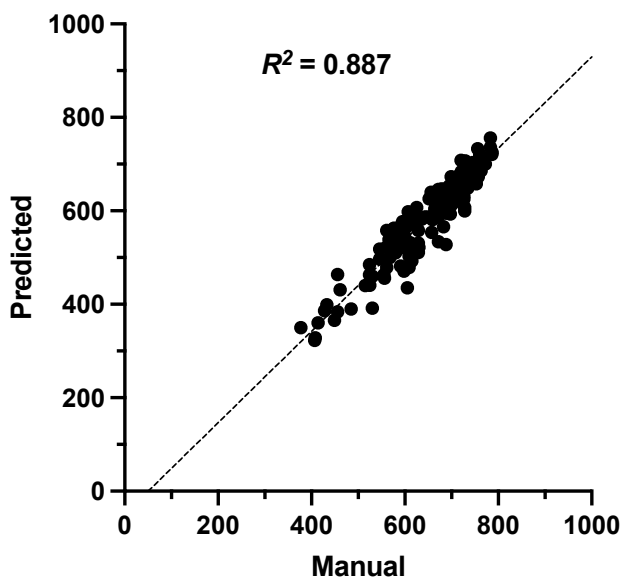

D

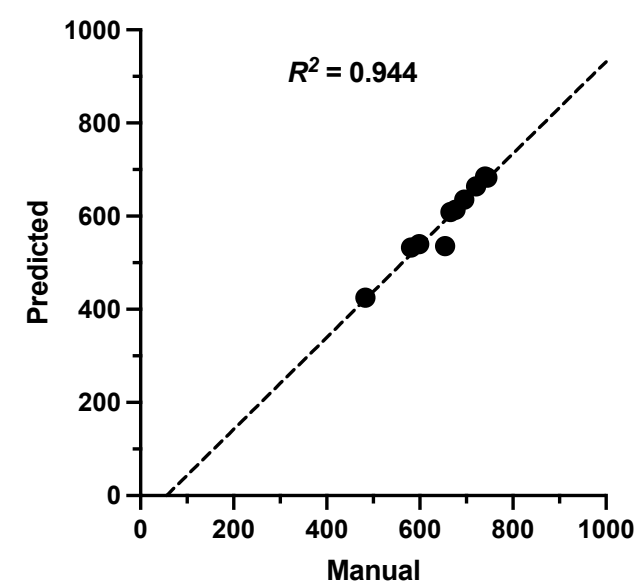

Fig. 4 
bioRxiv preprint doi: https://doi.org/10.1101/2021.10.25.465674; this version posted October 26, 2021. The copyright holder for this preprint (which was not certified by peer review) is the author/funder. All rights reserved. No reuse allowed without permission.
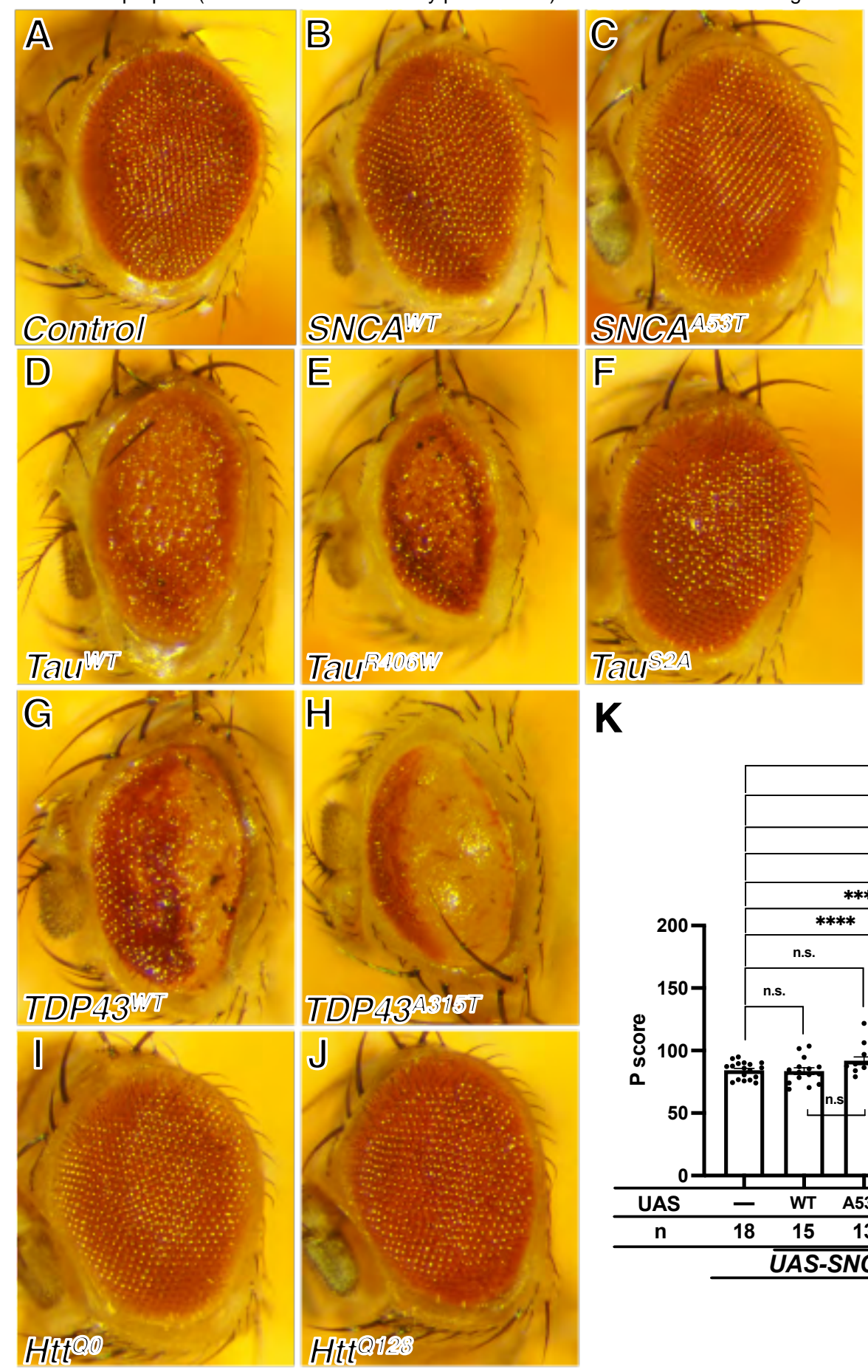

K

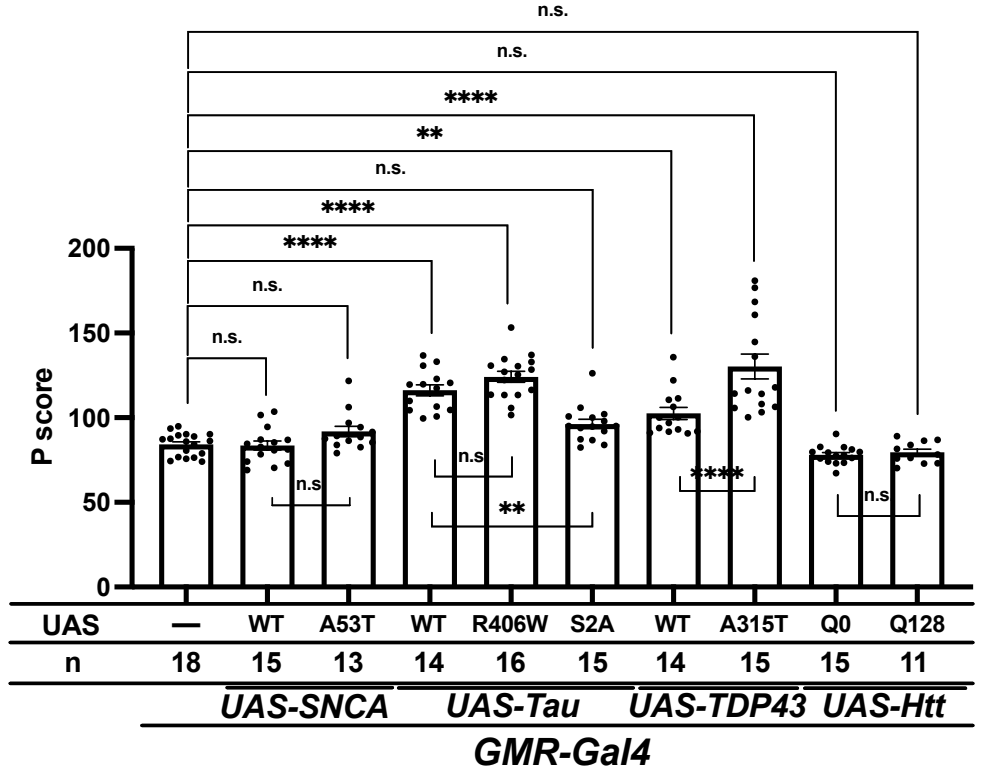

Fig. 5 